\title{
Acoustic Traps and Lattices for Electrons in Semiconductors
}

\author{
M. J. A. Schuetz, ${ }^{1,2}$ J. Knörzer, ${ }^{1}$ G. Giedke, ${ }^{3}$ L. M. K. Vandersypen, ${ }^{4}$ M. D. Lukin, ${ }^{2}$ and J. I. Cirac ${ }^{1}$ \\ ${ }^{1}$ Max-Planck-Institut für Quantenoptik, Hans-Kopfermann-Strasse 1, 85748 Garching, Germany \\ ${ }^{2}$ Physics Department, Harvard University, Cambridge, Massachusetts 02318, USA \\ ${ }^{3}$ Donostia International Physics Center, Paseo Manuel de Lardizabal 4, E-20018 San Sebastián, Spain \\ and Ikerbasque Foundation for Science, Maria Diaz de Haro 3, E-48013 Bilbao, Spain \\ ${ }^{4}$ Kavli Institute of NanoScience, TU Delft, P.O. Box 5046, 2600 GA Delft, Netherlands \\ (Received 23 May 2017; revised manuscript received 21 August 2017; published 24 October 2017)
}

\begin{abstract}
We propose and analyze a solid-state platform based on surface acoustic waves for trapping, cooling, and controlling (charged) particles, as well as the simulation of quantum many-body systems. We develop a general theoretical framework demonstrating the emergence of effective time-independent acoustic trapping potentials for particles in two- or one-dimensional structures. As our main example, we discuss in detail the generation and applications of a stationary, but movable, acoustic pseudolattice with lattice parameters that are reconfigurable in situ. We identify the relevant figures of merit, discuss potential experimental platforms for a faithful implementation of such an acoustic lattice, and provide estimates for typical system parameters. With a projected lattice spacing on the scale of $\sim 100 \mathrm{~nm}$, this approach allows for relatively large energy scales in the realization of fermionic Hubbard models, with the ultimate prospect of entering the low-temperature, strong interaction regime. Experimental imperfections as well as readout schemes are discussed.
\end{abstract}

DOI: 10.1103/PhysRevX.7.041019

Subject Areas: Condensed Matter Physics,

Quantum Physics, Quantum Information

\section{INTRODUCTION}

The ability to trap and control particles with the help of well-controlled electromagnetic fields has led to revolutionary advances in the fields of biology, condensed-matter physics, high-precision spectroscopy, and quantum information, enabling unprecedented control both in the study of isolated single particles as well as in fewand many-body systems subject to controlled and tunable interactions. Prominent examples range from using optical tweezers for probing the mechanical properties of DNA $[1,2]$ to the realizations of Bose-Einstein condensates [3-5] and numerous breakthrough investigations of strongly correlated quantum many-body systems with both trapped ions [6] and ultracold atoms in optical lattices [7,8]. At the same time, the ever-improving control of materials and fabrication of semiconductor nanostructures has led to a proliferation of quasiparticles in such systems and a quest to trap and isolate them in order to gain deeper insights into their properties and interactions. While quantum dots have been developed into excellent traps for charged and neutral quasiparticles and have contributed to a wealth of exciting insights [9], scaling them to the many-body regime remains

Published by the American Physical Society under the terms of the Creative Commons Attribution 4.0 International license. Further distribution of this work must maintain attribution to the author(s) and the published article's title, journal citation, and DOI. either a fabrication or operational challenge. This motivates our search for trapping mechanisms that bring the generality and flexibility of optical lattices to the solid-state setting.

While an optical approach may be feasible [10], surface acoustic waves (SAWs) have recently been used in a range of exciting experiments to trap electrons [11-15] or excitons [16] in moving potentials. When following this approach, however, particles are typically lost on a relatively fast time scale of $\sim 10 \mathrm{~ns}$, as a consequence of finite sample sizes and propagation speeds set by the speed of sound to $\sim 3 \times 10^{3} \mathrm{~m} / \mathrm{s}$. Inspired by these experiments, here we propose and analyze engineered stationary and quasistationary (movable) acoustic trapping potentials and acoustic lattices (ALs) as a generic strategy for trapping, cooling, and controlling quasiparticles as well as a potential on-chip, solid-state platform for the simulation of quantum many-body systems. While in this work we use the generation of an effective standing-wave lattice for electrons as the main example of our technique, our theoretical approach generalizes immediately to other trap configurations. In particular, focused SAWs [17] might allow for the generation of quasi zero-dimensional traps for electrons akin to optical tweezers, thereby entering a new parameter regime in the context of acoustic tweezers; so far, the latter have been used only in a high-temperature, classical regime to trap and manipulate microparticles immersed in fluids above the SAW-carrying solid [18].

Our basic scheme involves counterpropagating SAWs that are launched in opposite directions from two (or more) 
standard interdigital transducers (IDTs) $[19,20]$ patterned either directly onto a piezoelectric substrate such as GaAs or on some piezoelectric island, as demonstrated, for example, in Ref. [21]; for a schematic illustration, compare Fig. 1. Because of the intrinsic piezoelectric property of the material, the SAWs are accompanied by a (time-dependent) periodic electric potential and strain field, generating a well-controlled potential landscape (of the same spatial and temporal periodicity) for electrons confined in conventional quantum wells or purely twodimensional crystals such as transition-metal dichalcogenides (TMDCs), with a periodicity on the order of $\sim 100 \mathrm{~nm}$ for SAW frequencies of $\sim 20 \mathrm{GHz}$ [22]. Based on a perturbative Floquet approach, we show that the electron's potential landscape can effectively be described by a time-independent pseudolattice with a lattice spacing $\sim a=\lambda / 2$, provided that certain conditions are fulfilled (as specified below). Intuitively, the occurrence of such an effective time-independent potential can be understood from the fact that sufficiently heavy electrons cannot adiabatically follow a rapidly oscillating force as created by the SAW-induced electric potential; therefore, the electron will effectively be trapped close to the potential minimum if its wave function spreads slowly enough such that it is still close to its original position after one oscillation period of the SAW field. We identify the relevant figures of merit for this novel setup [cf. Eq. (2)] and show how the system parameters can be engineered and dynamically tuned. As a guideline for an experimental realization of the proposed setup, we derive a set of selfconsistency requirements that allows us to make clear predictions about the material properties needed for a

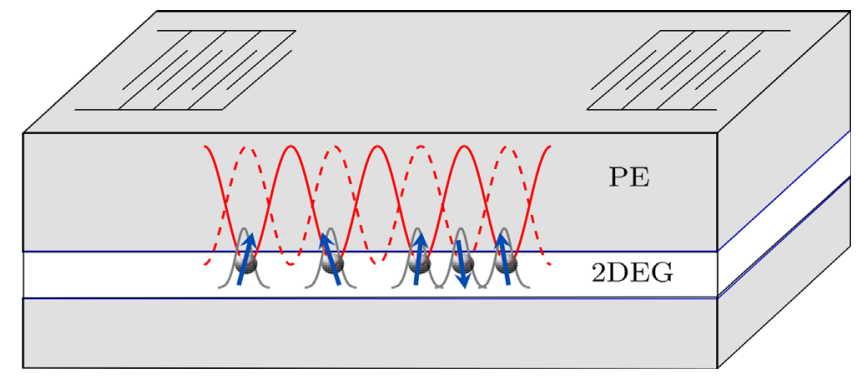

FIG. 1. Exemplary schematic illustration of the setup. In a piezoelectric solid (PE) counterpropagating SAWs (as induced by standard IDTs deposited on the surface $[19,20])$ generate a timedependent, periodic electric potential for electrons confined in a conventional two-dimensional electron gas (2DEG). If the SAW frequency $\omega / 2 \pi=v_{s} / \lambda$ is sufficiently high (as specified in the main text), the electron's potential landscape can effectively be described by a time-independent pseudolattice with a lattice spacing $a=\lambda / 2$. The potential depth (lattice spacing) can be controlled conveniently via the power (frequency) applied to the IDTs, while an additional screening layer (not shown) allows for tuning the strength of the Coulomb interaction between the particles [23]. In more complex structures, the setup can consist of multiple layers on top of some substrate. faithful implementation. Consequently, we identify strategies to meet these requirements with state-of-the-art experimental techniques and suitable material choices. Concerning the latter, we analyze the viability of different heterostructures with high effective electron masses which support high-velocity sound waves, e.g., AlN/diamond or, alternatively, TMDCs such as $\mathrm{MoS}_{2}$ or $\mathrm{WSe}_{2}$. While we discuss the relevant decoherence mechanisms as well as other relevant experimental imperfections for specific systems, the very basic principles of our approach should be of broad applicability to various physical solid-state platforms. In particular, thanks to the generic nature of our analysis and the variety of fields (strain, electric, magnetic) that potentially accompany SAWs, our framework is readily applicable to a broad class of (quasi)particles, including, for example, electrons, holes, trions, and excitons. While our theoretical treatment is (to some extent) reminiscent of trapped ions, allowing us to capitalize on ideas and results from this welldeveloped field of research, we show that the emergent effective dynamics can be captured by the Fermi-Hubbard model, very much like for fermionic ultracold atoms in optical lattices, albeit in unprecedented parameter regimes, because of ultrahigh charge-to-mass ratios and naturally long-ranged Coulomb interactions. Our approach provides an alternative to standard (gate-defined) quantum dots, providing a highly regular periodicity simply set by the SAW wavelength, with minimal fabrication requirements (without any further gate patterning), and the potential to deterministically move around the acoustically defined quantum dots by simply changing the phase of the excitation applied to the IDTs. Also, our trapped-ion-inspired pseudopotential approach makes our proposal significantly different from previous theoretical [23] and experimental investigations [16,21], where particles trapped inside a dynamic, moving $\mathrm{AL}$ (rather than a quasistationary, standing $\mathrm{AL}$, as considered here) are inevitably lost within a rather short time scale, $\sim 10$ ns.

\section{THEORETICAL FRAMEWORK}

In this section, we first develop a general theoretical framework describing particles in low-dimensional semiconductor structures in the presence of (SAW-induced) high-frequency standing waves. We employ both classical and quantum-mechanical tools in order to identify the relevant figures of merit and specify the conditions for the validity of our theoretical framework. The experimental feasibility of our scheme is discussed for specific setups in Sec. III.

Surface acoustic waves.-SAWs are phonon excitations that propagate elastically on the surface of a solid within a depth of roughly one wavelength $\lambda[19,20]$. In the case of a piezoelectric material, SAWs can be generated electrically based on standard interdigital transducers deposited on the surface, with a SAW amplitude proportional to the amplitude (square root of the power) applied to the IDTs 
$[13,19,20]$. Typically, such an IDT consists of two thin-film electrodes on a piezoelectric material, each formed by interdigitated fingers. Whenever a radio frequency signal is applied to such an IDT, a SAW is generated if the resonance condition $p=v_{s, \alpha} / f$ is met; here, $p, v_{s, \alpha}$, and $f=\omega / 2 \pi$ refer to the IDT period, the sound velocity of a particular SAW mode $\alpha$, and the applied frequency, respectively $[19,20,24]$. As evidenced by numerous experimental studies [25-28], SAWs can interact with a two-dimensional electron gas (2DEG) via the electric (and/or strain) field accompanying this elastic wave.

Classical analysis. - To illustrate our approach, let us first consider the classical dynamics of a single, charged particle of mass $m$ (also referred to as electron in the following) exposed to a SAW-induced monochromatic piezoelectric standing wave of the form $\phi(x, t)=\phi_{0} \cos (k x) \cos (\omega t)$. Here, $\omega=v_{s} k$ refers to the dispersion relation of a specific SAW mode, and the time-dependent potential experienced by the electron is $V(x, t)=e \phi(x, t)$ with an amplitude $V_{\mathrm{SAW}}=e \phi_{0}$ (where $e$ denotes the electron's charge). In the absence of a piezoelectric potential, a similar periodic potential derives from the (strain-induced) deformation potential associated with a SAW [16]; our theoretical analysis applies to both scenarios, as it is independent of the microscopic origin of the SAW-induced potential $V(x, t)=V_{\text {SAW }} \cos (k x) \cos (\omega t)$. While the motion in the $z$ direction is frozen out for experimentally relevant temperatures, a potential pattern of the same periodic form could be produced in the $y$ direction using appropriately aligned pairs of IDTs launching counterpropagating SAWs [23]. In this scenario the electron's motional degrees of freedom are separable into two one-dimensional problems of the same structure. Alternatively, using, for example, etching techniques or gate-defined structures as described in Refs. [13,14], effectively one-dimensional wires with strong transverse confinement in the $y$ direction may be considered. Therefore, in any case only the motion in the $x$ direction is discussed in the following. Then, in dimensionless units, where $\tilde{x}=k x$ and $\tau=\omega t / 2$, Newton's equation of motion for the electron's position $x(t)$ reads

$$
\frac{d^{2} \tilde{x}}{d \tau^{2}}+2 q \sin (\tilde{x}) \cos (2 \tau)=0
$$

where we introduce the (dimensionless) stability parameter $q=V_{\mathrm{SAW}} / E_{S}$, with the emerging energy scale

$$
E_{S}=m v_{s}^{2} / 2
$$

that is, the classical kinetic energy of a particle with mass $m$ and velocity equal to the speed of sound $v_{s}$ of the driven SAW mode; as we show below, the energy scale $E_{S}$ turns out to be a key figure of merit in our setup. In the Lamb-Dicke limit $\tilde{x} \ll 1$, Eq. (1) reduces to the so-called Mathieu equation [cf. Eq. (A1)], which is known to govern the dynamics of ions in Paul traps [29,30]. We assess the stability of the electron's motion against thermal noise by numerically solving Eq. (1), for initial conditions set as $\tilde{x}_{0}=0, \tilde{v}_{0}:=[d \tilde{x} / d \tau]_{\tau=0}=\sqrt{2 k_{B} T / E_{S}}$; here, according to $m v_{0}^{2} / 2=k_{B} T / 2$, the initial velocity $v_{0}$ is identified with the temperature $T$ by simple equipartition. Solutions to this problem are deemed stable if the maximal excursion $x_{\max }$ is smaller than one-half of the lattice spacing $\left(\tilde{x}_{\max }<\pi\right)$, even for very long time scales, and unstable otherwise. The results of this classification procedure are shown in Fig. 2: Stable (bounded) solutions can be found only for sufficiently low temperatures (with $k_{B} T \ll E_{S}$ ) and certain values of the stability parameter $q$. In particular, in the regime $q^{2} \ll 1$, $k_{B} T \ll E_{S}$ stable trajectories $\tilde{x}(\tau)$ consist of slow harmonic oscillations at the secular frequency $\omega_{0} / \omega \approx q / \sqrt{8}$, superimposed with fast, small-amplitude oscillations at the driving frequency $\omega$ (also referred to as micromotion [30]); compare Fig. 2(b). When neglecting the micromotion within the socalled pseudopotential approximation (as routinely done in the field of trapped ions [30]), the electron's (secular) dynamics is effectively described by that of a time-independent harmonic oscillator with (slow) frequency $\omega_{0} \ll \omega$; for further analytical and numerical details, see Appendix A.

Quantum-mechanical Floquet analysis.-The results described above can be corroborated within a fully quantum-mechanical model. Here, the electron's dynamics are governed by the time-dependent Hamiltonian

$$
H_{S}(t)=\frac{\hat{p}^{2}}{2 m}+V_{\mathrm{SAW}} \cos (\omega t) \cos (k \hat{x}),
$$

where $\hat{x}$ and $\hat{p}$ refer to the particle's position and momentum operators, respectively. The Hamiltonian $H_{S}(t)$
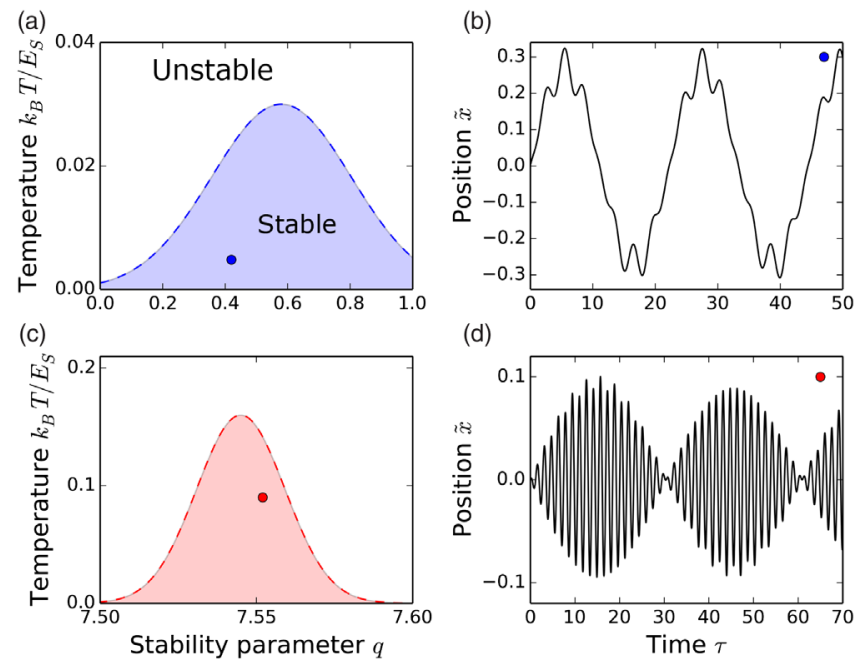

FIG. 2. Approximate stability diagrams of the classical equation of motion in the low- $q$ (upper plot) and high- $q$ (lower plot) regimes, respectively. The dots denote trajectories corresponding to some exemplarily chosen parameter sets $\left(q, k_{B} T / E_{S}\right)$. 
satisfies $H_{S}(t+T)=H_{S}(t)$ due to the time-periodic nature of the external driving, with $T=2 \pi / \omega$. In a high-frequency field, where the period of the force $T$ is small compared to all other relevant time scales, the particle's dynamics can be approximately described by a time-independent Hamiltonian $H_{\text {eff }}$. As detailed in Appendix B, $H_{\text {eff }}$ can be calculated in a systematic expansion in the inverse of the driving frequency $\omega[31,32]$. Then, up to second order in $\sim \omega^{-1}$, we obtain

$$
H_{\text {eff }}=\frac{\hat{p}^{2}}{2 m}+V_{0} \sin ^{2}(k \hat{x})
$$

where $V_{0}=\varepsilon^{2} E_{S}$, with the small parameter $\varepsilon=q / \sqrt{8}$. The second term $V_{\text {eff }}(\hat{x})=V_{0} \sin ^{2}(k \hat{x})$ demonstrates the formation of an effectively time-independent, spatially periodic acoustic lattice, with a lattice spacing $a=\lambda / 2=\pi / k$ and potential depth $V_{0}=\varepsilon^{2} E_{S}$. Similar to the case for trapped ions, lattice sites are found at the nodes of the timedependent force $\mathbf{F}(x, t) \sim \sin (k x) \cos (\omega t) \mathbf{x}$ associated with the potential $V(x, t)$. This force changes its sign on a time scale $\sim \omega^{-1}$; if this is fast compared to the particle's dynamics $\sim \omega_{0}^{-1}$, the particle will be dynamically trapped, because it does not have sufficient time to react to the periodic force before this force changes its sign again. Within the usual harmonic approximation, where $V_{\text {eff }}(\hat{x}) \approx(m / 2) \omega_{0}^{2} \hat{x}^{2}$, the effective trapping frequency $\omega_{0}$ can be estimated as $\omega_{0} / \omega \approx q / \sqrt{8}$, which coincides exactly with the (classical) result for the slow secular frequency $\omega_{0}$ in the pseudopotential regime (with $q^{2} \ll 1$ ). Accordingly, the AL can be rewritten as $V_{\text {eff }}(\hat{x})=\left(\omega_{0} / \omega\right)^{2} E_{S} \sin ^{2}(k \hat{x})$, with the first (perturbative) factor accounting for the inherent separation of time scales between the fast driving frequency $\omega$ and the slow secular frequency $\omega_{0}$. Written in this form, the effective acoustic potential $V_{\text {eff }}(\hat{x})$ is reminiscent of standard dipole traps for ultracold atoms. Here, the effective optical potential for a two-level system driven by a Rabi frequency $\Omega$ with detuning $\Delta$ in a electromagnetic standing wave takes on the form $V_{\text {opt }}(\hat{x})=\left(\Omega^{2} / 4 \Delta^{2}\right) \Delta \sin ^{2}(k \hat{x})$, with the selfconsistent requirement $\Delta \gg \Omega$. Therefore, with the prefactor $\sim \Omega^{2} / 4 \Delta^{2}$ being small for self-consistency, we can associate the role $E_{S}$ plays in the acoustical case with the role the detuning $\Delta$ plays in the optical setting. Along these lines, for robust trapping it is favorable to increase the materialspecific quantity $E_{S}$, thereby achieving a larger trap depth $V_{0}$ while keeping both the stability parameter $q=V_{\mathrm{SAW}} / E_{S}$ and thus also the perturbative parameter $\varepsilon$ constant. This can be well understood intuitively, since trapping due to a rapidly oscillating (SAW) field becomes possible only if the particle is too inert to adiabatically follow the periodically applied force: an electron does not significantly move away from a potential minimum if during one oscillation period of the SAW field its wave function spreads slowly enough such that it is still close to its original position when the minimum reforms. This simplified (pseudopotential) picture is valid for relatively heavy electrons with high mass $m$ and sufficiently high driving frequency (that is, high speed of sound $v_{s}$ ), as captured by an elevated sound energy $E_{S}=(m / 2) v_{s}^{2}$.

Cooling in the presence of micromotion.-While our previous discussion focuses exclusively on the timedependent system's dynamics, in the following we extend our studies and introduce a dissipative model, which describes the electron's motional coupling to the (thermal) phonon reservoir. For details of the derivation, see Appendix C. Within one unified Born-Markov and Floquet framework, we derive an effective quantum master equation for the electronic motion in the vicinity of one lattice site, fully taking into account the explicit time dependence of the system Hamiltonian Eq. (3). Since the quantum-state evolution due to this quantum master equation is Gaussian, one can readily derive a closed set of equations for the first- and second-order moments of the position and momentum observables; formally, it takes on the form $\dot{\mathbf{v}}=\mathcal{M}(t) \mathbf{v}+\mathbf{C}(t)$, with $\mathbf{v}=\left(\langle\hat{x}\rangle_{t},\langle\hat{p}\rangle_{t},\left\langle\hat{x}^{2}\right\rangle_{t}\right.$, $\left.\left\langle\hat{p}^{2}\right\rangle_{t},\langle\hat{x} \hat{p}+\hat{p} \hat{x}\rangle_{t}\right)^{\top}$. This equation of motion can be readily solved by numerical integration; a prototypical result of this procedure is displayed in Fig. 3. In the regime $q^{2} \ll 1$, our numerical findings show that (i) the electronic motion can be described very well by a simple damped harmonic oscillator with secular frequency $\omega_{0}$, (ii) the electronic motion is cooled by the phonon reservoir, and (iii) the Lamb-Dicke approximation is well satisfied. Let us elaborate on these statements in some more detail. (i) As evidenced by the dashed red line in Fig. 3, we find that the effective, time-independent master equation,

$$
\begin{aligned}
\dot{\rho}= & -i \omega_{0}\left[a^{\dagger} a, \rho\right]+\gamma\left(\bar{n}_{\mathrm{th}}\left(\omega_{0}\right)+1\right) \mathcal{D}[a] \rho \\
& +\gamma \bar{n}_{\mathrm{th}}\left(\omega_{0}\right) \mathcal{D}\left[a^{\dagger}\right] \rho,
\end{aligned}
$$

captures very well the most pertinent features of the electronic dynamics (for $q^{2} \ll 1$ ). Here, $\gamma$ is the effective, incoherent damping rate due to coupling to the thermal phonon reservoir, $\bar{n}_{\text {th }}\left(\omega_{0}\right)=1 /\left(\exp \left[\hbar \omega_{0} / k_{B} T\right]-1\right)$ gives the thermal occupation number of the phonon bath at frequency $\omega_{0}, \mathcal{D}[a] \rho=a \rho a^{\dagger}-(1 / 2)\left\{a^{\dagger} a, \rho\right\}$ denotes the standard dissipator of Lindblad form, and $a^{(\dagger)}$ refers to the usual annihilation (creation) operators for the canonical harmonic oscillator. As a consequence of the presence of the dissipator, the first-order moments $\langle\hat{x}\rangle_{t},\langle\hat{p}\rangle_{t}$ decay towards zero in the asymptotic limit $t \rightarrow \infty$. However, the second-order moments retain the periodicity of the external driving for arbitrarily long times (with a periodicity $\bar{T}=\omega T / 2=\pi$ ), which is the signature of an emerging quasistationary state (cf. Appendix C for details) and the persisting micromotion which manifests itself in the fast oscillating dynamics of the position and momentum variances, as depicted in the inset of Fig. 3. (ii) As suggested by our analytical results (cf. Appendix C for details), the phonon reservoir provides an efficient cooling 


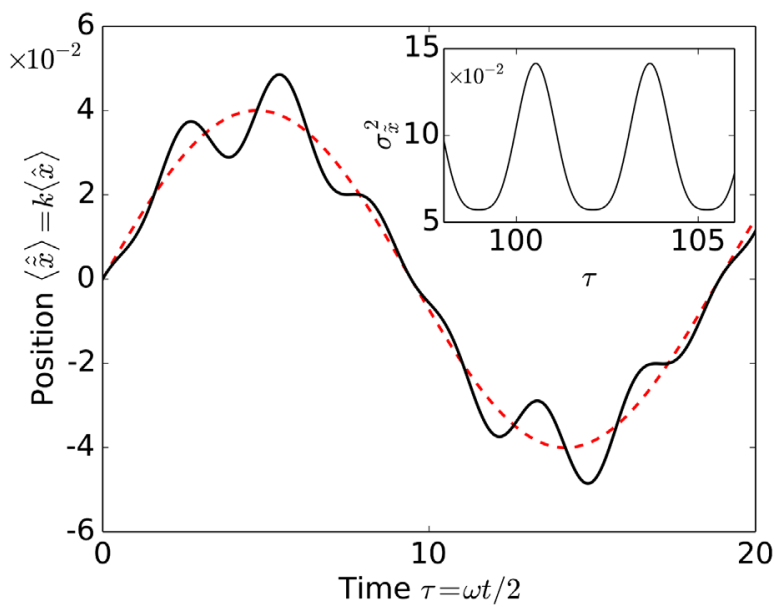

FIG. 3. Exact numerical simulation [based on Eqs. (C37) and (5)] for the electron's trajectory $\langle\hat{x}\rangle_{t}$ (solid black line), showing a slow secular motion with frequency $\omega_{0}$ that is superimposed by fast, small-amplitude micromotion oscillations. When disregarding micromotion, the dynamics can approximately be described by a simple damped harmonic oscillator with secular frequency $\omega_{0}$ (dashed red line). The initial state has been set as a coherent state with $\langle\hat{\tilde{x}}\rangle=0,\langle\hat{\tilde{p}}\rangle=0.01$. Other numerical parameters are $q=0.47, \gamma / \omega_{0}=10^{-3}, k_{B} T / \hbar \omega_{0}=10^{-1}, \omega_{0} / \omega \approx 0.17$. Inset: Position variance $\sigma_{\tilde{x}}^{2}=\left\langle\hat{\tilde{x}}^{2}\right\rangle-\langle\hat{\tilde{x}}\rangle^{2}$ at times when transient effects have decayed.

mechanism for the electron provided that the host temperature is sufficiently low, that is, $k_{B} T \ll \hbar \omega_{0}$. The influence of the electronic micromotion on this cooling mechanism can be condensed in the following statement: in the pseudopotential regime (for which $q^{2} \ll 1$ ), the expectation value for the averaged quantum kinetic energy (over one micromotion period) features a surplus of energy, in addition to the zero-point kinetic energy in the ground state of $\hbar \omega_{0} / 4$. This excess energy $\Delta_{\text {heat }} \gtrsim \hbar \omega_{0} / 4$ may be viewed as micromotion-induced heating and amounts to merely a factor of 2 increase only in the particle's timeaveraged kinetic energy [33]. These results are explicated in greater detail in Appendix C. (iii) We have numerically verified that both the expectation value for the electron's motion as well as the corresponding fluctuations are small compared to the SAW wavelength $\lambda=2 \pi / k$, i.e., $k\langle\hat{x}\rangle_{t} \ll 1$ and $k \sigma_{x} \ll 1$, with $\sigma_{x}^{2}=\left\langle\hat{x}^{2}\right\rangle_{t}-\langle\hat{x}\rangle_{t}^{2}$, thereby justifying our Lamb-Dicke approximation [with $\cos (k \hat{x}) \approx$ $\left.\mathbb{1}-\left(k^{2} / 2\right) \hat{x}^{2}\right]$ self-consistently.

Self-consistency requirements.-Our theoretical framework is valid provided that the following conditions are satisfied. (i) First, the Markov approximation holds given that autocorrelations of the bath (which typically decay on a time scale $\sim \hbar / k_{B} T$ ) decay quasi-instantaneously on the time scale of system correlations $\sim \gamma^{-1}$ [34]. In principle, the damping rate $\gamma$ should be replaced by the thermally enhanced rate $\gamma_{\text {eff }}=\gamma\left[\bar{n}_{\mathrm{th}}\left(\omega_{0}\right)+1\right]$; however, we are mostly interested in the low-temperature, pseudopotential regime where $\gamma_{\text {eff }} \approx \gamma$. Thus, the Markov approximation yields the condition $\hbar \gamma \ll k_{B} T$. (ii) Second, the (weakcoupling) Born approximation holds provided that the dissipative damping rate $\gamma$ is small compared to the relevant system's transition frequencies, yielding the requirement $\gamma \ll \omega_{0}$. In the low- $q$ limit, taking conditions (i) and (ii), together with the prerequisite for efficient ground-state cooling, $k_{B} T \ll \hbar \omega_{0}$, yields the chain of inequalities $\hbar \gamma \ll k_{B} T \ll \hbar \omega_{0}$. In this regime, the weak-coupling Born approximation $\left(\gamma \ll \omega_{0}\right)$ is satisfied very well. (iii) Third, the characteristic separation of time scales between the (slow) secular motion and the (fast) micromotion, with $\omega_{0}=\varepsilon \omega$ and $\varepsilon=q / 2 \sqrt{2} \ll 1$, gives the requirement $\omega_{0} \ll \omega$. (iv) Fourth, the energy scale $\hbar \omega$ has to be much smaller than $E_{S}$ in order to ensure the existence of at least one bound state per lattice site; the latter can be estimated as $n_{b}=V_{0} / \hbar \omega_{0}=\varepsilon E_{S} / \hbar \omega=$ $(\varepsilon / 2) m v_{s} / \hbar k$, leading to $\hbar \omega \ll E_{S}$ in the regime $\varepsilon \ll 1$, $n_{b} \gtrsim 1$. Note that the existence of at least one bound state per lattice site $\left(n_{b} \gtrsim 1\right)$ may always be fulfilled by choosing the lattice spacing $a=\pi / k$ sufficiently large, at the expense of more severe temperature requirements for ground-state cooling and smaller energy scales in the emerging Hubbard model (see below). Finally, the parameter regime of interest can be condensed into one line of inequalities as $(\hbar=1)$

$$
\gamma \ll k_{B} T \ll \omega_{0} \ll \omega \ll E_{S} .
$$

Let us discuss the implications of Eq. (6) in more detail. (i) In the parameter regime described by Eq. (6) the acoustic trap is stable against thermal fluctuations, because $k_{B} T \ll$ $V_{0}$ with $V_{0}=\varepsilon^{2} E_{S}$; in other words, $V_{0}=n_{b} \omega_{0} \gg k_{B} T$, if $\omega_{0} \gg k_{B} T$ and $n_{b} \gtrsim 1$, as desired. The condition $k_{B} T \ll \omega_{0}$, however, may be relaxed if ground-state cooling is not necessarily required, akin to the physics of optical tweezers. In this case, the less stringent condition $V_{0} \gg k_{B} T$ still ensures a thermally stable trap. (ii) The self-consistency requirement $\gamma \ll k_{B} T$ derives from the Markov assumption of having a short correlation time of the phonon bath $\gamma \tau_{c} \ll 1$, with $\tau_{c} \sim 1 / k_{B} T$. However, in the lowtemperature regime, the correlation time $\tau_{c}$ may as well be set by the bandwidth of the bath $\Delta_{B}$ (that is, the frequency range over which the bath at hand couples to the system), rather than just temperature. In that case, one may drop the condition $\gamma \ll k_{B} T$, leading to a slightly refined regime of interest with $\gamma, k_{B} T \ll \omega_{0} \ll \omega \ll E_{S}$, provided that the Markov assumption $\gamma \tau_{c} \ll 1$ is still satisfied with $\tau_{c} \sim \Delta_{B}^{-1}$. (iii) As a direct consequence of the presence of Mathieu-type instabilities, the proposed setup operates at relatively low SAW-induced amplitudes set by the energy scale $E_{S}$, with the potential amplitude due to a single IDT given as $V_{\mathrm{IDT}}=V_{\mathrm{SAW}} / 2=(q / 2) E_{S}<E_{S}$.

Again, Eq. (6) underlines a remarkably close connection to the established field of trapped ions, where (as a direct 
consequence of Mathieu's equation, just as in our setting) the inherent separation of time scales $\left(\omega_{0} \ll \omega\right)$ between (slow) secular motion and (fast) micromotion is well known, albeit at very different energy scales with typical driving frequencies $\omega / 2 \pi \sim 100 \mathrm{kHz}-100 \mathrm{MHz}$ [30]. Beyond this close analogy, our work identifies the importance of the energy scale $E_{S}=(m / 2) v_{s}^{2}$ in the proposed solid-state, SAW-based setting, as displayed by Eq. (6). Moreover, the first two inequalities in Eq. (6) derive directly from the intrinsic solidstate cooling mechanism provided by the phonon bath, whereas ions are typically cooled down to the motional ground state using laser-cooling techniques that (as opposed to our solid-state approach) explicitly involve the ion's internal level structure [30].

In the following, we address the experimental implications of the requirements listed in Eq. (6) for realistic setups and show how some of the conditions may in fact be relaxed.

\section{IMPLEMENTATION: HOW TO MEET THE REQUIREMENTS}

Our previous conceptual analysis reveals a specific set of requirements [as summarized in Eq. (6)] that should be fulfilled in order to ensure a faithful implementation of the proposed AL setup in an actual experiment. In the following we discuss several practical strategies in order to meet these conditions. Thereafter, we address several practical considerations that might be relevant under realistic experimental conditions.

Requirements.-First, rough (potentially optimistic, see below) estimates for the spontaneous emission rate of acoustic phonons $\sim \gamma$ may be inferred from low-temperature experiments on charge qubits in (GaAs) double quantum dots which indicate rates as low as $\gamma / 2 \pi \gtrsim 20 \mathrm{MHz}$ $(\hbar \gamma \gtrsim 0.1 \mu \mathrm{eV})$ [35-38]. We consider this estimate for the relaxation rate $\sim \gamma$ to be an optimistic but still adequate ballpark value for our SAW-induced acoustic traps, because the typical (i) temperatures [T (20-100) $\mathrm{mK}]$, (ii) length scales ( $\sim 300 \mathrm{~nm}$ for the dot-to-dot distance), (iii) transition frequencies ( $\sim \mathrm{GHz}$ in Ref. [37]), and (iv) host materials (GaAs) studied in Refs. [35-38] are all compatible with our setup. Furthermore, in Ref. [37] an Ohmic spectral density has been assumed (just like in our theoretical model discussed above) in order to fit the experimental data with the (thermally enhanced) decoherence rate $\gamma_{\text {eff }}=$ $\gamma\left[2 \bar{n}_{\text {th }}\left(\omega_{0}\right)+1\right]$, yielding $\gamma=\zeta \omega_{0}$ with the fit parameter $\zeta=(\pi / 4) \times 0.03 \sim 2.35 \times 10^{-2}$. Second, we consider typical dilution-fridge temperatures in the range of $T \sim$ (10-100) $\mathrm{mK}$ [corresponding to $k_{B} T \sim(1-10) \mu \mathrm{eV}$ ] [39]. For $\gamma / 2 \pi \approx 20 \mathrm{MHz}$, the first inequality in Eq. (6) is then safely satisfied even for the lowest temperatures under consideration $\left(k_{B} \times 10 \mathrm{mK} / 2 \pi \sim 200 \mathrm{MHz}\right)$. Still, since $\gamma$ varies significantly with both energy and length scales, phonon relaxation rates of $\gamma / 2 \pi \approx 20 \mathrm{MHz}$ for GaAs-based systems may be overly optimistic. In this case, operation at higher temperatures [in order to satisfy Eq. (6)] may still be avoided by employing (for example) phonon band gaps, as discussed in Ref. [35], or different materials such as silicon $[24,40]$, where the corresponding phonon-induced relaxation rates are much smaller [41], as a consequence of a much smaller electron-phonon coupling strength. All other things being equal, the SAW-induced potential depth $V_{\text {SAW }}$ will be reduced as well in a siliconbased setup, which, however, can be compensated by simply applying a larger rf power to the IDTs. Lastly, recall that the spontaneous emission rate $\gamma$ may be as large as $\gamma \approx k_{B} T$ and still be fully compatible with the desired regime of interest, if the correlation time of the phonon bath is set by (for example) the bandwidth $\Delta_{B}$ rather than temperature. Third, for high SAW frequencies, $\omega / 2 \pi \approx$ $25 \mathrm{GHz}$ [22], the energy $\hbar \omega \approx 100 \mu \mathrm{eV}$ yields a trapping frequency $\hbar \omega_{0} \lesssim 20 \mu \mathrm{eV}\left(q^{2} \ll 1\right)$. Altogether, we thus conclude that Eq. (6) can be satisfied with state-of-the-art experimental setups, provided that the material-specific energy scale $E_{S}$ is much larger than $\hbar \omega \approx 100 \mu \mathrm{eV}$. For electrons in standard GaAs and the lowest Rayleigh mode, however, we find $E_{S} \approx 2 \mu \mathrm{eV}$. In the following, we identify three potential, complementary strategies to solve this problem.

(1) Material engineering.-Our first approach involves sophisticated material engineering, with the aim to crank up the energy scale $E_{S}$. Here, we can identify three general, complementary strategies to increase the sound energy [cf. Eq. (2)]. (i) First, the effective mass $m$ crucially depends on both (a) the type of particle and (b) the host material. (a) Heavy holes or composite quasiparticles such as trions (also known as charged excitons) typically feature much higher effective masses than electrons in GaAs. (b) Compared to standard GaAs, where the effective electron mass is $m \approx 0.067 m_{0}$ ( $m_{0}$ refers to the freeelectron mass), in $\mathrm{Si} / \mathrm{SiGe}$ structures $m \approx 0.2 m_{0}$, while for electrons (heavy holes) in AlN $m \approx 0.33 m_{0}$ $\left(m_{h h}=3.89 m_{0}\right)$. (ii) Second, following common practice in the quest for SAW devices operating at ultrahigh frequencies [42-44], $v_{s}$ can be effectively increased by employing a specialized heterostructure involving, for example, diamond (which features the highest speed of sound). (iii) Third, the speed of sound $v_{s, \alpha}=\omega_{\alpha} / k$ can be enhanced even further by exciting higher-order Rayleigh modes $(\alpha>1)$ in the sample at the same wavelength [19]. In particular, layered half-space structures (such as AlN/ diamond, with $h$ denoting the thickness of the piezoelectric AlN layer) support so-called pseudosurface acoustic waves (PSAWs) propagating with exponential attenuation due to wave energy leakage into the bulk, in contrast to regular (undamped) SAWs $[19,45,46]$. As shown both theoretically and experimentally [45,46], this leakage loss can, however, become vanishingly small for certain magic film-thicknessto-wavelength ratios $h / \lambda$, such that for all practical purposes this PSAW mode can be seen as a true SAW mode 
which propagates with negligible attenuation. While SAWs by definition may not exceed the shear wave velocity $c_{s}$ ( $c_{s} \approx 12.32 \mathrm{~km} / \mathrm{s}$ for diamond) in the lower half-space, PSAW velocities can be significantly larger than $c_{s}$ and reach values of up to $v_{s} \approx 18 \mathrm{~km} / \mathrm{s}[45,46]$, that is about $40 \%$ higher than those of regular SAWs [46] and about a factor of $\sim 3.2$ higher as compared to the lowest Rayleigh mode for a homogeneous AlN half-space where $v_{s} \approx$ $5.6 \mathrm{~km} / \mathrm{s}$ [45]. Lastly, even higher velocities may be achieved if leakage losses into the bulk are suppressed when using freely suspended two-dimensional electron gases [47] rather than a (quasi-)semi-infinite half-space.

We verify these considerations using numerical finiteelement calculations, performed with the software package COMSOL [48] for GaAs/diamond (AlN/diamond) heterostructures; our simulations indeed show that the effective speed of sound can be significantly scaled up in comparison with the standard values in GaAs (AlN) [49]. In Fig. 4, the behavior of $v_{s}$ as a function of the width $h$ of the GaAs (AIN) layer is displayed. The results show both the second Rayleigh SAW modes in GaAs/diamond and AlN/ diamond, respectively, as well as one particular PSAW mode (as identified previously in Ref. [45]). For large $h$, the second Rayleigh SAW modes coincide with the corresponding second modes in the raw materials GaAs and AlN (without a diamond layer), as expected. On the other hand, in the limit of comparatively small $h \approx(50-200) \mathrm{nm}$, the SAW velocities are significantly larger compared to the first and second Rayleigh modes in pure GaAs (AlN), while for the PSAW mode $v_{s} \approx 18 \mathrm{~km} / \mathrm{s}$ at $h / \lambda \approx 0.57$. Moreover, in the case of piezoelectric coupling, the electric potential that accompanies the SAW has to be nonzero at the 2DEG, which is located somewhere in the center of the top GaAs (AlN) layer. As shown in Fig. 4, such configurations do exist in GaAs/diamond (AlN/diamond) heterostructures, while reaching the parameter regime $k_{B} T=1 \mu \mathrm{eV} \lesssim\left(10^{-3}-10^{-2}\right) E_{S}$. Hence, when suitably combining strategies (i)-(iii), we predict the feasibility of reaching $E_{S} \gtrsim 1 \mathrm{meV}$, which is sufficiently large to safely satisfy condition Eq. (6), as desired. Consider, for example, a two-dimensional hole gas at a AlN/GaN interface on top of diamond; here, the effective heavy-hole mass of GaN amounts to $m \approx 1.1 m_{0}$. When driving the PSAW mode identified in Fig. 4 , we find $E_{S} \approx 1.0 \mathrm{meV}$. Alternatively, we may consider monolayer transition-metal dichalcogenides such as $\mathrm{MoS}_{2}$ or $\mathrm{WSe}_{2}$, on top of some high-speed material such as diamond. While all TMDCs are piezoelectric due to the lack of inversion symmetry [51], some of them show relatively large effective masses; for example, the effective electron and hole mass in $\mathrm{MoS}_{2}$ amount to approximately $m \approx 0.67 m_{0}$ and $m \approx 0.6 m_{0}$, respectively [52,53]. Then, for electrons (charged trions) in $\mathrm{MoS}_{2}$ with effective mass $m \approx 0.67 m_{0}\left(m \approx 1.9 m_{0}\right)$ [53], as experimentally investigated, for example, in Refs. [54,55], and a diamond-boosted speed of sound

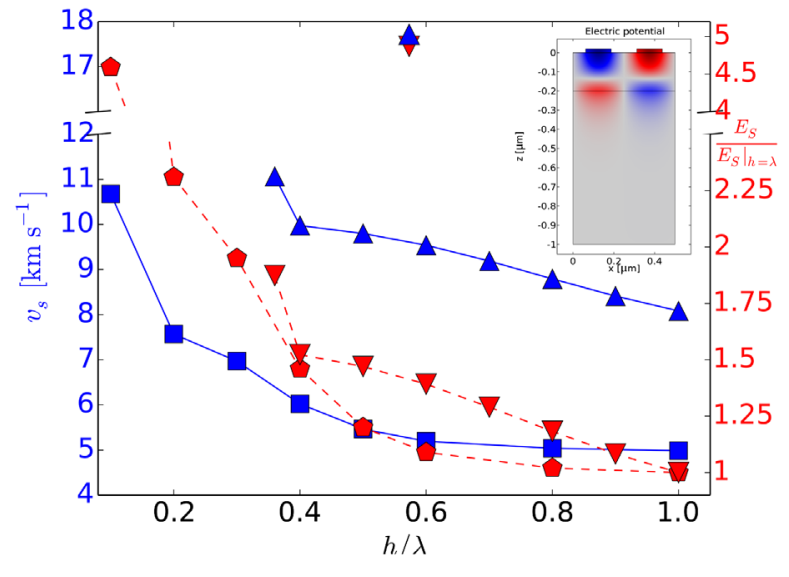

FIG. 4. Speed of sound $v_{s}$ (left axis) and kinetic sound energy $E_{S}$ normalized to its value at $h=\lambda$ (right axis) in layered heterostructures made of gallium arsenide (aluminum nitride) and diamond. All results are given as a function of $h$, which denotes the thickness of the GaAs (AIN) layer. Results for the second SAW modes and heavy holes are shown. Squares and pentagons (triangles) denote the numerical results for a GaAs/ diamond (AlN/diamond) heterostructure. $E_{S}(h=\lambda) \approx 32 \mu \mathrm{eV}$ for GaAs/diamond ( $\approx 205 \mu \mathrm{eV}$ for AlN/diamond). The data points are connected by lines to guide the eye. The isolated data points at $h \approx 0.57 \lambda$ denote an ultrahigh velocity PSAW mode in AlN/diamond (cf. Ref. [45]). Inset: Distribution of the piezoelectric potential at $f=12.2 \mathrm{GHz}$ of a second SAW mode for a layer thickness of $h=0.2 \mu \mathrm{m}$ in a GaAs/diamond heterostructure. The IDT finger spacing, hence the SAW wavelength, is set to be $\lambda=500 \mathrm{~nm}$. The results are obtained with the software package COMSOL.

$v_{s} \approx 18 \mathrm{~km} / \mathrm{s}$, we estimate $E_{S}$ to be as large as $E_{S} \approx$ $617 \mu \mathrm{eV}\left(E_{S} \approx 1.78 \mathrm{meV}\right)$. Further estimates of this type for different physical setups are summarized in Table I. Here, we cover the most relevant material properties for the implementation of the proposed AL setups only, whereas the interplay of different material-design strategies (i)-(iii) leads to an intricate problem involving various parameters (such as piezoelectric properties and the

TABLE I. Estimates for the energy scale $E_{S}$ for different physical setups. Examples marked with $*$ refer to the lowest SAW mode in GaAs, whereas those marked with $* *$ refer to relatively fast (diamond-boosted) values of $v_{s}$ in diamond-based heterostructures featuring high-frequency SAW and PSAW modes as investigated in Refs. $[45,46]$. Further details are given in the text.

\begin{tabular}{llcc}
\hline \hline Setup & $m / m_{0}$ & $v_{s}[\mathrm{~km} / \mathrm{s}]$ & $E_{S}[\mu \mathrm{eV}]$ \\
\hline Electrons in GaAs* & 0.067 & $\sim 3$ & $\sim 1.7$ \\
Heavy holes in GaAs** & 0.45 & $\sim(12-18)$ & $\sim 184-415$ \\
Electrons in $\mathrm{Si}^{* *}$ & 0.2 & $\sim(12-18)$ & $\sim 82-184$ \\
Holes in $\mathrm{GaN}^{* *}$ & 1.1 & $\sim(12-18)$ & $\sim 450-1010$ \\
Electrons in $\mathrm{MoS}_{2}{ }^{* *}$ & 0.67 & $\sim(12-18)$ & $\sim 274-617$ \\
Trions in $\mathrm{MoS}_{2}{ }^{* *}$ & 1.9 & $\sim(12-18)$ & $\sim 794-1787$ \\
\hline \hline
\end{tabular}


electron mobility), which we cannot cover in its full depth within the scope of this work.

While this material-engineering-based approach is fully compatible with our general theoretical framework, as described in Sec. II, in the following we present two additional schemes that allow for thermally stable trapping, at potentially higher temperatures than what we have found so far, but at the expense of a more involved theoretical description [which, however, is not necessarily restricted to the parameter regime given in Eq. (6)]; here, similarly to Sec. II, we first present a classical analysis of the dynamics, whereas a detailed, quantum-mechanical analysis thereof goes beyond the scope of this work and will be subject to future research.

(2) Exotic stability regions. - In the context of ion traps where stability is governed by the Mathieu equation [cf. Eq. (A1)], ion motion is stable in the primary stability region $\left(a_{\mathrm{dc}}=0,0<q<0.908\right)$ and then becomes unstable as $q$ is increased [30]. Stable motion, however, reoccurs at higher $q$ values, which we refer to as exotic stability regions in the following; these exotic stability regions were studied to some extent in the context of ion traps [56,57]. Here, we propose, as a second strategy to meet the selfconsistency requirements, to extend the previously established classical stability analysis to the next higher-lying $\left(a_{\mathrm{dc}}=0,7.5 \lesssim q \lesssim 7.6\right)$ stability region of the Mathieu equation. As evidenced in Fig. 2(d), in this high- $q$ regime, a separation between secular and fast (micro)motion is no longer possible. However, while the theoretical description of the dynamics becomes more involved, still the particles are found to be dynamically trapped, already at temperatures much higher than what we find in the low- $q$ regime. While $k_{B} T \lesssim 0.03 E_{S}$ for small $q$, in the high- $q$ regime (with $7.5<q<7.6$ ) thermal stability sets in already at $k_{B} T \lesssim 0.15 E_{S}$, thus alleviating temperature requirements by about an order of magnitude; cf. Fig. 2(c).

(3) Optimized driving schemes.-As a third strategy, we suggest to utilize polychromatic driving schemes, rather than the simple monochromatic driving considered so far. Recently, it has been experimentally demonstrated that such polychromatic drivings can eventuate arbitrary SAW wave fronts [58], thus allowing us to consider more general equations of motion of the form $\ddot{x}+f(\tau) x=0$, with some particular time dependence $f(\tau)$. For example, instead of the Mathieu equation for which $f(\tau)=2 q \cos (2 \tau)$ (no dc contribution), a simple two-tone driving scheme can be used to expand the stability regions, as previously suggested in Ref. [59]. Our numerical studies suggest that the superposition of higher harmonics in the form of $f(\tau)=$ $2 q\left[c_{1} \cos (2 \tau)+c_{2} \cos (4 \tau)+\cdots\right]$ may already enhance the robustness of the stability region in Fig. 2(a) against temperature by a factor of 2 , as compared to the standard Mathieu equation.

Technical considerations.-We now address several technical considerations that might be relevant for a faithful experimental realization of our proposal: (i) Since the potential amplitude due to a single IDT is limited by Mathieu-type stability arguments as $V_{\text {IDT }}=V_{\text {SAW }} / 2=$ $(q / 2) E_{S} \lesssim 0.5 \mathrm{meV}$ [60], the proposed setup operates at SAW-induced amplitudes that are about 2 orders of magnitude smaller than what is common for SAW-induced electron transport experiments (where typically $V_{\mathrm{IDT}} \approx$ $40 \mathrm{meV}[13,61])$. Note that this comparatively low driving amplitude amounts to a fraction of typical quantum dot charging energies. Today, quantum dots are routinely pulsed with similarly high amplitudes, and yet excellent charge and spin coherence is seen in experiments [62-64]. (ii) In a similar vein, as a direct consequence of the lowamplitude external drive, potential microwave-induced heating effects of the sample should be small. Furthermore, undesired heating may be suppressed efficiently by placing the IDTs very far away from the center of the trap, without losing acoustic power, thereby avoiding local heat dissipation near the center of the trap due to the applied rf power; for further details, see Appendix D. (iii) Minimization of cross-talk-related effects can be accomplished based on various techniques [65]: these can involve, for example, very careful choice of metalpackaging structure and dimensions, the judicious placement of ground connections to avoid ground loop effects, and the placement of thin metal-film ground strips between the IDTs. Moreover, because of the vast difference between the speed of light $\left(c \approx 10^{8} \mathrm{~m} / \mathrm{s}\right)$ and the speed of sound $\left(v_{s} \approx 10^{4} \mathrm{~m} / \mathrm{s}\right)$, for a given frequency the wavelength associated with the electromagnetic (EM) cross talk is about 4 orders of magnitude larger than the SAW wavelength (even when accounting for the refractive index of the specific material), and therefore practically flat on the relevant length scale of a few lattice sites; for $\omega / 2 \pi \approx 30 \mathrm{GHz}$, the wavelength is in the millimeter range, i.e., much larger than the acoustic lattice spacing $a=v_{s} /(\omega / \pi) \approx 170 \mathrm{~nm}$.

\section{APPLICATIONS}

The possibility to acoustically trap charged particles in a semiconductor environment should open up many experimental possibilities, well beyond the scope of this work. Here, we briefly describe just two potential exemplary applications; see also our discussion in the final section of this paper.

\section{A. Mobile acoustic quantum dots}

By adiabatically tuning the phases applied to the IDTs, one may displace the AL in both the $x$ and $y$ direction, thereby creating mobile acoustic quantum dots, with the possibility to transfer in this way quantum information stored in the spin degree of freedom of the particle; for a schematic illustration, see Fig. 5. Here, in contrast to standard SAW-based mobile quantum dots $[13,14]$, the 


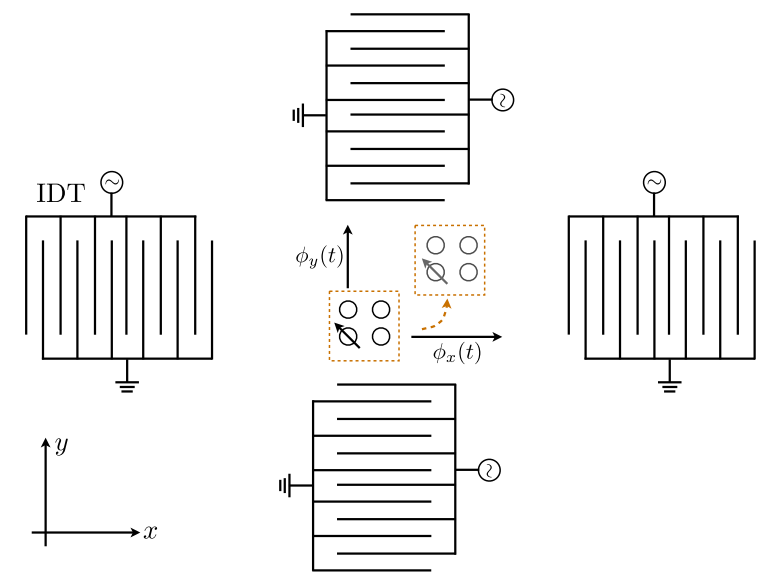

FIG. 5. Schematic illustration (top view, not to scale) for a quasistationary two-dimensional AL which can be controllably displaced in both $x$ and $y$ direction by adiabatically tuning the phases applied to the IDTs. The dashed (orange) box highlights a small sublattice consisting of just four lattice sites, before and after the adiabatic ramp.

speed $v_{\text {eff }}$ at which the trapped particles can be moved around between different locations in the 2DEG would not simply be set by the SAW's speed of sound $v_{s}$, but could rather be controlled in situ by the time derivative of the phases applied to the IDTs, with an upper bound roughly given by the adiabaticity condition $\left(\varepsilon_{\text {ad }} \ll 1\right)$ as $v_{\text {eff }}=\varepsilon_{\text {ad }} a\left(\omega_{0} / 2 \pi\right) \lesssim 100 \mathrm{~m} / \mathrm{s}$. Apart from thermal fluctuations, the trapping lifetime in such a mobile quantum dot will be limited by tunnel coupling to neighboring mobile quantum dots inside the AL (while the spin lifetime remains unaffected for spin-coherent tunneling); as we show in more detail below, this coupling can be suppressed controllably by going to a larger SAW wavelength $\lambda$, at the expense of more stringent ground-state cooling requirements as the level spacing $\omega_{0}$ decreases. For a mobile AL with near unit filling, however, tunneling is largely suppressed due to Coulomb blockade effects and the (spin) dynamics is governed by the next lower energy scale (the exchange coupling), as we discuss and quantify next.

\section{B. Towards many-body physics}

Many-body physics.-While our previous discussion has exclusively focused on dynamically trapping and cooling single particles in SAW-induced potentials, in our second example we provide a simple characterization of our setup for the potential investigation of quantum many-body systems. We show that (at dilution-fridge temperatures) our system can be naturally described by an extended Anderson-Hubbard model, with the ultimate prospect of entering the low-temperature, strong interaction regime where $k_{B} T \ll t<U$; here, $t$ and $U$ refer to the standard hopping and interaction parameters of the Hubbard model, as we specify below. We provide estimates for these quantities in terms of the relevant parameters characterizing the $\mathrm{AL}$, and show how they can be engineered and (dynamically) tuned. For this analysis, again we restrict ourselves to the pseudopotential regime $\left(\omega_{0} \ll \omega\right)$, where the effects of the fast, small-amplitude micromotion on the Hubbard parameters $t, U$ can be neglected. Thereafter, we discuss several approaches that may be used in order to detect and accurately probe the resulting quantum phases of matter.

Estimates for Hubbard parameters.-Consider an ensemble of fermionic charged particles inside a periodic one- or two-dimensional AL, with roughly one particle per site (corresponding to electron densities $\sim 10^{10} \mathrm{~cm}^{-2}$ for a two-dimensional AL with $a \sim 100 \mathrm{~nm}$ ). If all energy scales involved in the system dynamics are small compared to the excitation energy to the second band $\sim \hbar \omega_{0}$ (for example, $k_{B} T \ll \hbar \omega_{0}$, as required for ground-state cooling), the electrons will be confined to the lowest Bloch band of the AL, and the system can effectively be described by the extended Anderson-Hubbard Hamiltonian [23,66-68]

$$
\begin{aligned}
H_{\mathrm{AFH}}= & -t \sum_{\langle i, j\rangle, \sigma}\left(c_{i, \sigma}^{\dagger} c_{j, \sigma}+\text { H.c. }\right)+\sum_{i} \mu_{i} n_{i} \\
& +\sum_{\sigma, \sigma^{\prime}} \sum_{i j k l} U_{i j k l} l_{i, \sigma^{\prime}}^{\dagger} c_{j, \sigma}^{\dagger} c_{k, \sigma} c_{l, \sigma^{\prime}},
\end{aligned}
$$

where the fermionic operator $c_{i, \sigma}\left(c_{i, \sigma}^{\dagger}\right)$ annihilates (creates) an electron with spin $\sigma=\uparrow, \downarrow$ at site $i ; n_{i, \sigma}=c_{i, \sigma}^{\dagger} c_{i, \sigma}$ and $n_{i}=n_{i, \uparrow}+n_{i, \downarrow}$ refer to the spin-resolved and total occupation number operators, respectively. In Eq. (7), we retain the nearest-neighbor hopping term only, as specified by a tunneling amplitude $t$, but account for the full effect of the repulsive (long-range) Coulomb interactions $\sim U_{i j k l}$. The remaining (second) term, with a variable on-site energy $\mu_{i}$, acts like a spatially varying chemical potential and describes potential disorder effects (as we discuss in more detail below). In the limit of homogeneous on-site energies with $\mu_{i}=$ const, Eq. (7) reduces to the minimal Hubbard model, if all but the largest on-site interaction terms are neglected (with $U_{i i i i}=U$ in standard notation). In the limit $V_{0} \gg E_{R}$ (where $E_{R}=\hbar^{2} k^{2} / 2 m$ is the recoil energy), the tunneling rate $t$ is given by $t / E_{R} \approx$ $(4 / \sqrt{\pi})\left(V_{0} / E_{R}\right)^{3 / 4} \exp \left[-2 \sqrt{V_{0} / E_{R}}\right]$ [7], setting the upper limit $t<E_{R}$. In terms of the relevant AL parameters, this relation can be rewritten as

$$
t / E_{S} \approx\left(2 \sqrt{2 \pi n_{b}}\right)^{-1} q^{2} \exp \left[-4 n_{b}\right],
$$

showing that the tunneling rate $t \sim q^{2}$ can be tuned via the stability parameter $q$, reaching at maximum $t / E_{S} \lesssim$ $3 \times 10^{-3}$ within the lowest stability region for (fixed) $n_{b} \gtrsim 1$; here, the existence of at least one bound state $\left(n_{b} \gtrsim 1\right)$ ensures both $V_{0} / E_{R}=4 n_{b}^{2} \gtrsim 4$ and $t \ll \hbar \omega_{0}$, as required. Therefore, with $E_{R} / E_{S}=\varepsilon^{2} /\left(4 n_{b}^{2}\right)$, we find $t<E_{R} \ll E_{S}$ such that realistically $E_{S} \gg 300 \mu \mathrm{eV}$ is 
required in order to access the coherent many-body regime where $t \gg k_{B} T$, at dilution-fridge temperatures. Making use of the strategies outlined in the previous section, this regime seems to lie within reach of state-of-the-art experimental capabilities. The order of magnitude for the Coulomb integral $U_{i j k l}$ can be roughly estimated as $U_{i j k l} \sim$ $e^{2} / 4 \pi \epsilon a$ (where $\epsilon$ denotes the effective dielectric constant of the material). Since $t \sim E_{R} \sim a^{-2}$ and $U_{i j k l} \sim 1 / a$, the relative importance of the hopping term $\sim t$ as compared to the Coulomb interactions can be conveniently controlled via the SAW frequency $\omega=\pi v_{s} / a$ [23]. Taking (for example) $a \sim 300 \mathrm{~nm}$, this rough estimate yields $U_{i j k l} \sim$ $380 \mu \mathrm{eV}$ (for GaAs, where $\epsilon \approx 12.5 \epsilon_{0}$ ), which exceeds any realistic hopping amplitude $t$ by far, but also violates the assumptions underlying the model Hamiltonian Eq. (7). To enter a parameter regime where the simplified toy model Eq. (7) becomes applicable, special heterostructures with a metallic screening layer close to the 2DEG may be employed, while in a similar vein the thickness of the spacer layer (separating the 2DEG from the $\delta$-doping layer) may also be reduced in favor of increased screening effects $[23,39]$. In this scenario, a simple image-charge-based estimate shows that the Coulomb interaction is reduced by a factor $f_{\text {scr }} \approx 1-\left[1+4(d / a)^{2}\right]^{-1 / 2}$ (where $d$ refers to the distance between the 2DEG and the metallic screening plate), while retaining its $\sim 1 / r$ scaling [23]. Accordingly, the estimate quoted above reduces from $\sim 380 \mu \mathrm{eV}$ for $d \rightarrow \infty$ down to $\sim 50 \mu \mathrm{eV}$ for $d \sim 0.3 a \sim 90 \mathrm{~nm}$. As we discuss in more detail below, this approach does not only allow for tuning the strength of the Coulomb interaction (albeit not in situ), but at the same time reduces the detrimental effects due to background impurities [39]. In a regime where the latter is negligible, the next lower energy scale is set by the exchange coupling $J=4 t^{2} / U$, which describes effective spin-spin interactions via virtual hopping processes in the regime $U \gg t$. With the Coulomb interaction reduced to $U \approx 10 t$, the regime $t \gg k_{B} T$ (and, therefore, $J \approx t / 2 \gg k_{B} T$ ) should then give access to experimental studies of quantum magnetism [7]. For a comprehensive overview of the key quantities of our analysis and self-consistent estimates thereof, see Appendix D. In Appendix D we also discuss relevant electron spin decoherence effects which may compete with the observation of coherent spin physics.

Detection schemes. - In order to measure the resulting collective many-body state in an actual experiment, several approaches may be available. (i) First, the electron excitation spectrum could be probed using inelastic light scattering, as has been done experimentally in a closely related setup (based on electrons confined in etched pillars in a high-quality GaAs quantum well with mobility $\mu \sim 3 \times 10^{6} \mathrm{~cm}^{2} / \mathrm{Vs}$ ) in Ref. [69]. (ii) Second, transport measurements, in which a small dc voltage $V_{\mathrm{dc}}$ is applied across the AL, should carry signatures of the phase of the Hubbard model in the resulting dc current; compare, for example, Refs. [23,70,71]. The corresponding dc current $I_{\mathrm{dc}}$ will be blocked in the Mott-insulator regime, whereas Ohm's law $I_{\mathrm{dc}} \propto V_{\mathrm{dc}}$ should hold in a metallic phase $[23,72]$. (iii) Third, charge-imaging methods could also be used to demonstrate regular carrier localization in the acoustic lattice, somewhat similar to the detection of single electrons trapped by impurity centers [73,74]. (iv) Fourth, capacitance spectroscopy techniques (as demonstrated, for example, in Ref. [75]) could be used in order to measure the density of states by detecting the ability to tunnel in from a backplane. (v) Fifth, optical readout of the charge and spin state could be achieved with methods developed for selfassembled quantum dots [76], in particular in TMDC-based setups $[51,54,55,77]$. Similar to self-assembled quantum dots, our SAW-defined quantum dots and lattices trap both electrons and holes at the same location and could thus support quantum dot excitons and trions. The charge- and spin-dependent interaction with quasiresonant light fields can be used for readout via resonance fluorescence [78] or the Kerr effect $[79,80]$. Moreover, it is conceivable that related optical techniques for state preparation and spin rotation can be adapted as well. Note that, due to the expected homogeneity of our SAW-generated lattice sites, we also expect largely identical optical spectra across the lattice which may facilitate global readout and collective optical effects. (vi) Lastly, apart from these well-established measurement techniques, we propose to perform local siteresolved detection by adiabatically changing the phases at the IDTs $\phi(t)$ and then loading one lattice site after the other (very much like in a CCD camera) into nearby gatedefined quantum dots, where both the charge as well as the spin degree of freedom could be measured via wellestablished spin-to-charge conversion techniques [81].

\section{EFFECTS OF DISORDER}

Disorder in the AL will affect the (Anderson) Hubbard model, as described by the second term in Eq. (7), where (in the presence of disorder) $\mu_{i}$ is essentially a randomly fluctuating variable. In a semiconductor the dominant source of disorder is due to charged impurities, which includes both (i) desired contributions (such as the dopants used for forming the 2DEG) as well as (ii) undesired ones due to bulk or surface impurities [39]. While the dominant source of disorder (i) due to remote donor scattering can be largely removed in structures with a relatively large spacer thickness $\sim 85 \mathrm{~nm}[39,82]$, the second one (ii) has been identified as the main mechanism limiting the mobility $\mu$ in ultraclean 2DEGs $[83,85]$. Still, as experimentally demonstrated in Ref. [85], mobilities exceeding $\sim 10^{7} \mathrm{~cm}^{2} / \mathrm{V} \mathrm{s}$ can be realized for dilution-fridge temperatures $T \sim 100 \mathrm{mK}$, resulting in a mean free path $l_{\mathrm{mfp}}=$ $\mu v_{F} m / e$ of up to $l_{\mathrm{mfp}} \sim 120 \mu \mathrm{m}$ (here, $v_{F}$ refers to the Fermi velocity [23]). In the low-density regime of interest with $n_{\mathrm{el}} \sim 10^{10} \mathrm{~cm}^{-2}$ (corresponding to half-filling for a 
lattice spacing of $a \sim 100 \mathrm{~nm}$ ) the mean free path is expected to drop to $l_{\mathrm{mfp}} \sim 15 \mu \mathrm{m}$ [85], which is still much larger than the lattice spacing $a \sim 100 \mathrm{~nm}$. To further compensate for residual disorder originating from background impurities (ii), one may resort to special heterostructures with a conducting backplane, as suggested in Ref. [39]. Also, in periodic arrays of quantum dots, signatures of Hofstadter's butterfly [86] have been observed at high magnetic fields [70,71], as a result of the interplay between the periodic potential and quantized Hall orbitals, suggesting that disorder from the substrate can in fact be sufficiently small to investigate coherent lattice physics. This discussion certainly provides the basis for some optimism, but a dedicated research program (rather than just a literature survey) may be required to fully understand and characterize the role of disorder in this system; compare Ref. [72] for recent efforts in this direction based on gate-defined lattices in GaAs. While the effect of disorder on the single-particle level is well understood [87], the intricate interplay between interactions and disorder in the Hubbard model (as studied in Refs. [88-91]) yields a nontrivial regime in its own right that may be explored systematically in the proposed setup by deliberately controlling the amount of disorder.

\section{SUMMARY AND OUTLOOK}

In summary, we propose and analyze the formation of an all-solid-state acoustic lattice with a highly regular periodicity set by the SAW wavelength (without any further gate patterning). We develop a theoretical framework reminiscent of trapped-ion physics thus connecting two previously unrelated fields of research. With this framework at our disposal, we identify the relevant figures of merit for this system and discuss potential experimental platforms for a faithful implementation of such acoustic lattices, with the ultimate potential to study yet unexplored parameter regimes, thanks to specific system properties such as ultralight particle masses, intrinsic electron-phonon cooling, and strong interparticle interactions. Here, let us emphasize again the flexibility (and generic nature) of the proposed scheme: SAWs exist in many materials (semiconductor heterostructures, TMDCs) and can be endowed with a variety of accompanying fields (depending on the material used: strain, electric, magnetic) and superposed to different standing-wave patterns. Therefore, the proposed scheme should be applicable to a variety of different (quasi) particles and allow one to study different lattice geometries.

Finally, we highlight possible directions of research going beyond our present work. (i) While we focus on a simple square-lattice geometry, more sophisticated lattice geometries might be explored, given the design flexibilities associated with SAW devices [19]. (ii) For simplicity, in this work we disregard the potential presence of magnetic fields and/or spin-orbit effects, which stem from the underlying material properties. Therefore, without any further sophisticated engineering, these additional ingredients could be readily implemented, giving rise to rich phase diagrams and, for example, the formation of topological quantum spin Hall states [92]. Finally, we may envisage several setups that are complementary to the system we study in this work. (iii) Acoustic lattices for dipoles. Our ideas can be generalized towards an acoustic lattice for solid-state dipoles (rather than charged particles), e.g., for indirect excitons, which consist of electrons and holes from two different parallel quantum well layers, thereby complementing previous experimental studies on SAW-induced lattices for exciton polaritons both in moving [21] and standing-wave [93] configurations in the regime of many particles per lattice site. As evidenced by several experiments (where the repulsive character of the interaction shows up as a positive and monotonic line shift with increasing density [94]), indirect excitons behave as effective dipoles perpendicular to the plane $[95,96]$. Because of the spatial separation between the electron and hole layers in this coupled quantum well structure, the intrinsic radiative lifetimes of optically active indirect excitons exceeds that of their direct counterparts by orders of magnitude and can be in the range of several microseconds [96]. In TMDC-based setups our approach may be used to dynamically trap and to spatially and spectrally isolate single excitons, thereby complementing experiments based on static strain engineering [97]. (iv) Acoustic lattices for ions. The electric potential (created and controlled at the surface) due to standard IDTs extends into the material, but also into the vacuum above the surface [98]. In principle, this should allow for the integration of our SAW-based setup with ions above the surface that are exposed to this acoustically induced electric potential, leading to new hybrid setups and complementing other approaches towards regular, disorder-free surface traps for ions in which the lattice spacing is simply set by the SAW wavelength. With comparatively large parameter values for $E_{S}(\approx 420 \mathrm{meV}$ for Be ions on top of GaAs), preliminary estimates show that a pseudopotential trap depth of several $\mathrm{meV}$ should be possible within the lowest stability region (where $q^{2} \ll 1$ ), provided that the ion can be stabilized in the direction normal to the surface within the SAW wavelength. (v) Magnetic lattices. While the acoustic lattice described above is based on coupling to the particle's external motional degree of freedom (as is the case with Paul traps for ions), in closer analogy to optical lattices for ultracold atoms, SAWs in piezomagnetic materials such as Terfenol-D [99-101] may be used in order to couple to the particle's internal spin degree of freedom, thereby inducing a spatially inhomogeneous Stark shift on the electron's spin resonance which will act as an external potential for the electron's motion [102]. In this setup, for a fixed detuning of the ESR driving frequency from the Zeeman splitting, the effective trap depth can (in principle) be made arbitrarily large, provided that sufficient SAW power is available. 
In conclusion, this discussion indicates that by combining the control and flexibility of SAWs with the rich variety of material properties of heterostructures, the emerging field of quantum acoustics opens a large number of further research directions with the ultimate goal of understanding the behavior of correlated electrons in technologically relevant materials and molecules and building a universal quantum simulator.

\section{ACKNOWLEDGMENTS}

M. J. A. S. would like to thank the Max-Kade foundation for financial support and MPQ for hospitality. M. J. A. S., J. K., and J. I. C. acknowledge support by the DFG within the Cluster of Excellence NIM. G. G. acknowledges support by the Spanish Ministerio de Economáa y Competitividad through the Project No. FIS2014-55987P and thanks MPQ for hospitality. L. M. K. V. acknowledges support by a European Research Council Synergy grant and the Netherlands Organization for Scientific Research (NWO). Work at Harvard was supported by NSF, Center for Ultracold Atoms, CIQM, Vannevar Bush Fellowship, and AFOSR MURI. M. J. A. S. and J. K. thank Alejandro Gonzalez-Tudela, Kristiaan De Greve, Hans Huebl, Eric Kessler, Hubert Krenner, Florian Marquardt, Javier Sanchez-Yamagishi, Paulo Santos, Robert Ukropec, Mathias Weiler, Dominik Wild, Susanne Yelin, and Peter Zoller for fruitful discussions.

M. J. A. S. and J. K. contributed equally to this work.

\section{APPENDIX A: CLASSICAL STABILITY ANALYSIS}

\section{Mathieu equation}

Performing a Taylor expansion for the electric field close to the origin, $\sin (\tilde{x}) \approx \tilde{x}$, Eq. (1) can be mapped onto the well-known Mathieu differential equation by identifying the parameters appearing in the standard Mathieu differential equation,

$$
\frac{d^{2} \tilde{x}}{d \tau^{2}}+\left[a_{\mathrm{dc}}+2 q \cos (2 \tau)\right] \tilde{x}=0,
$$

as $a_{\mathrm{dc}}=0$ (no de voltage) and $q=V_{\mathrm{SAW}} / E_{S}$.

In the case of vanishing dc contribution, according to Ref. [29], there is a stability zone for $0<q<q_{\max }$, with $q_{\max } \approx 0.92$, resulting in the maximum potential depth of $V_{\mathrm{SAW}}=q_{\max } E_{S}$. The lowest-order approximation to the ion trajectory $x(t)$ in the case $q^{2} \ll 1$ is found to be

$$
x(t) \approx 2 A C_{0} \underbrace{\cos \left(\beta \frac{\omega}{2} t\right)}_{\text {secular }} \underbrace{\left[1-\frac{q}{2} \cos (\omega t)\right]}_{\text {micromotion }},
$$

where $\beta \approx q / \sqrt{2}$. If the fast low-amplitude oscillations contained in the second factor are neglected, the secular motion can be approximated by that of a harmonic oscillator with frequency $\omega_{0}=\beta \omega / 2 \ll \omega$. The condition for the lowest-order approximation $q^{2} \ll 1$ is equivalent to a separation of time scales between secular and micromotion, that is, $\omega_{0} \ll \omega$. In this regime, the dynamics can be described by an effective pseudopotential.

\section{Classical pseudopotential}

The classical dynamics in a high-frequency field can be described by an effective time-independent Hamiltonian. Following Refs. [31,32], it can be calculated in a systematic expansion in the inverse of the frequency $\omega$. If the period of the force is small compared to the other time scales of the problem, it is possible to separate the motion of the particle into slow and fast parts. This simplification is due to the fact that the particle does not have sufficient time to react to the periodic force before this force changes its sign. Based on this separation of time scales, the motion for the slow part is computed explicitly up to the order $\omega^{-4}$. Note that the effective time-independent Hamiltonian depends on a coordinate $X(t)$ which describes the slow part of the motion; this coordinate is not the location of the particle, even though they are almost identical at high frequencies $\omega$. As outlined in Refs. [31,32], the decomposition of $x(t)$ into slow and fast components can be written as

$$
x(t)=X(t)+\xi(X, \dot{X}, \omega t),
$$

where the fast part of the motion $\xi$ fulfills

$$
\bar{\xi}=\frac{1}{2 \pi} \int_{0}^{2 \pi} d \tau \xi(X, \dot{X}, \tau)=0 .
$$

By expanding $\xi$ in powers of $1 / \omega$,

$$
\xi=\sum_{i=1}^{\infty} \frac{1}{\omega_{i}} \xi_{i}
$$

such that Eq. (A1) leads to an equation for $X$ that is time independent, and following Refs. [31,32], we find the following (classical) effective Hamiltonian describing the slow dynamics $X(t)$ :

$$
\begin{aligned}
H_{\text {eff }}= & \frac{P^{2}}{2 m}\left[1+\frac{3}{8} q^{2} \cos ^{2}(k X)\right] \\
& +\frac{q}{8} V_{\mathrm{SAW}} \sin ^{2}(k X)+\mathcal{O}\left(\omega^{-5}\right) .
\end{aligned}
$$

Here, $P$ is the momentum conjugate to $X$. Given a solution $X(t)$, the solution of the original problem can be obtained to appropriate order of $1 / \omega$ since $\xi$ is known explicitly in terms of $X[31,32]$. The pseudopotential for the average motion of the electron, $V_{\text {eff }}=V_{0} \sin ^{2}(k X)$, with an amplitude given by 


$$
V_{0}=\frac{q}{8} V_{\mathrm{SAW}}=\frac{q^{2}}{8} E_{S}
$$

is also referred to as ponderomotive potential [30]. Note that the correction to the kinetic term in Eq. (A6) is a fourth-order term, while the pseudopotential $V_{\text {eff }}$ is a second-order contribution in $1 / \omega$. Close to the origin $x=0$, the effective potential $V_{\text {eff }}$ can be approximated by a harmonic potential $V_{\text {eff }}(x)=(m / 2) \omega_{0}^{2} x^{2}$ with an oscillation frequency $\omega_{0}=(q / \sqrt{8}) \omega$, which is equivalent to the result obtained above from the Mathieu equation. Using this definition of the trapping frequency, the ponderomotive potential becomes

$$
V_{\text {eff }}=\left(\frac{\omega_{0}}{\omega}\right)^{2} E_{S} \sin ^{2}(k X) .
$$

We can then estimate the number of bound states $n_{b}$ as

$$
n_{b} \approx \frac{V_{0}}{\hbar \omega_{0}}=\frac{1}{2} \sqrt{\frac{V_{0}}{E_{R}}},
$$

with the recoil energy $E_{R}=\hbar k^{2} / 2 m$.

\section{Stability diagrams}

Here, we provide further details on our classical stability analysis. First, we note that the stability diagrams shown in Sec. II are of approximate character as they were obtained by interpolating our numerical results. This is due to the deliberate choice of defining a stable trajectory in terms of the maximal excursion during a sufficiently long propagation time: two trajectories with almost equal parameters $q$ and $k_{B} T / E_{S}$ can be judged as stable and unstable by this definition, respectively, because only one of their amplitudes exceeds the cutoff value set to one-half of the lattice constant $(a / 2)$. Second, the notion of (thermal) stability may be defined via the mean free path as well, by taking $l_{\mathrm{mfp}}$ as our cutoff value, in contrast to the trapping condition $\tilde{x}_{\max }<\pi$. In that case, the regions of thermal stability increase as compared to the ones shown in the main text, provided that $l_{\mathrm{mfp}}>a / 2$. The last inequality is likely to be fulfilled in high-mobility 2DEGs where $l_{\mathrm{mfp}} \sim 10 \mu \mathrm{m}$. Third, the stability analysis underlying Fig. 2 neglects damping in the classical equation of motion; incorporating an additional friction term may alter the notion of stability, since particles that escape one lattice site can then be dynamically trapped at a different lattice site. Lastly, the state initialization via equipartition of thermal and kinetic energies describes an average condition; in practice, only a fraction of the electrons will fulfill this condition, where the details depend on the statistical distribution of the initial conditions. In order to estimate the statistical fraction of electrons whose (initial) velocity $v$ is smaller than $v_{0}=\sqrt{k_{B} T / m}$, given by equipartition of thermal and kinetic energies of the particle, we assume a MaxwellBoltzmann distribution of velocities,

$$
p(v) d v=2 \sqrt{\frac{m}{2 \pi k_{B} T}} \exp \left(-\frac{m v^{2}}{2 k_{B} T}\right) d v,
$$

which yields $\int_{0}^{v_{0}} p(v) d v \approx 0.68$; i.e., given a thermal ensemble of particles, we find that a significant fraction of the electrons is found to be trapped.

\section{APPENDIX B: QUANTUM-MECHANICAL FLOQUET ANALYSIS}

Preliminaries.-We consider a quantum system with a Hamiltonian that is periodic in time, $H(t+T)=H(t)$. Floquet theory provides a natural framework to treat such a system $[31,32]$. The Bloch-Floquet theorem states that the eigenstates of the Schrödinger equation,

$$
i \frac{\partial}{\partial t}|\Psi\rangle=H|\Psi\rangle
$$

obey the form

$$
\left|\Psi_{\lambda}\right\rangle=e^{-i \lambda t}\left|u_{\lambda}(\omega t)\right\rangle
$$

where $u_{\lambda}$ are periodic with respect to $\omega t$ with period $2 \pi$; that is, $u_{\lambda}[x, \omega(t+T)]=u_{\lambda}(x, \omega t)$, with $\omega=2 \pi / T$. The states $u_{\lambda}$ are called Floquet states and $\lambda$ is the so-called quasienergy. They have a natural separation into a slow part $e^{-i \lambda t}$ (with the natural choice $0 \leq \lambda<\omega$ ) and a fast part $u_{\lambda}(x, \omega t)$. Now, the goal is to find an effective description for the slow part of the dynamics as was done above for the classical dynamics. Formally, this is done by introducing a gauge transformation,

$$
|\phi\rangle=e^{i F(t)}|\Psi\rangle
$$

where $F(t)$ is a Hermitian operator that is a periodic function of time $t$, with the same period as $H(t)$, such that the effective Hamiltonian $H_{\text {eff }}$ in the Schrödinger equation,

$$
\begin{gathered}
i \frac{\partial}{\partial t}|\phi\rangle=H_{\mathrm{eff}}|\phi\rangle \\
H_{\mathrm{eff}}=e^{i F} H e^{-i F}+i\left(\frac{\partial}{\partial t} e^{i F}\right) e^{-i F},
\end{gathered}
$$

is time independent. In particular, $H_{\text {eff }}$ can then be used to predict trapping due to oscillating potentials [31].

Typically, $F$ and $H_{\text {eff }}$ cannot be computed exactly. Following Refs. [31,32], we expand $H_{\text {eff }}$ and $F$ in powers of $1 / \omega$ and choose $F$ such that $H_{\text {eff }}$ is time independent to any given order. In the following, we compute the effective Hamiltonian, 


$$
H_{\mathrm{eff}}=\sum_{n} \frac{1}{\omega^{n}} H_{\mathrm{eff}}^{(n)},
$$

explicitly up to fourth order in $1 / \omega$.

\section{Second order}

Given the temporal periodicity of the driving only, it has been shown [31,32] that the odd terms $H_{\text {eff }}^{(1)}, H_{\text {eff }}^{(3)}$ from the perturbative expansion Eq. (B6) vanish. Hence, the leadingorder term (besides the purely kinetic contribution $p^{2} / 2 m$ ) of the effective Hamiltonian is of second order in $1 / \omega$.

For the single-particle Hamiltonian under consideration,

$$
H(t)=\frac{\hat{p}^{2}}{2 m}+V_{\mathrm{SAW}} \cos (\omega \mathrm{t}) \cos (k \hat{x}),
$$

up to second order in $1 / \omega$ we find

$$
H_{\text {eff }}=\frac{\hat{p}^{2}}{2 m}+\frac{q}{8} V_{\mathrm{SAW}} \sin ^{2}(k \hat{x}),
$$

which is the second-order result given in Eq. (4). Hence, similar to the classical treatment, also within the quantummechanical Floquet framework, the effective potential, which is of second order in the dimensionless coefficient $\omega_{0} / \omega$, can be written as

$$
V_{\text {eff }}(\hat{x})=\left(\frac{\omega_{0}}{\omega}\right)^{2} E_{S} \sin ^{2}(k \hat{x}) .
$$

Leading-order corrections to this result are of the order $\mathcal{O}\left(\omega^{-4}\right)$.

\section{Fourth order}

Computing Eq. (B6) explicitly up to $\mathcal{O}\left(\omega^{-4}\right)$ yields

$$
\begin{aligned}
H_{\mathrm{eff}}= & \frac{\hat{p}^{2}}{2 m}+\frac{q}{8} V_{\mathrm{SAW}} \sin ^{2}(k \hat{x}) \\
& +\frac{1}{2 m}\left[\hat{p}^{2} g(\hat{x})+2 \hat{p} g(\hat{x}) \hat{p}+g(\hat{x}) \hat{p}^{2}\right] \\
& +\frac{q^{2}}{32} E_{R} \sin ^{2}(k \hat{x})+\mathcal{O}\left(\omega^{-5}\right),
\end{aligned}
$$

where

$$
g(\hat{x})=\frac{3}{32} q^{2} \cos ^{2}(k \hat{x}) .
$$

In the classical limit (where $\hat{x}$ and $\hat{p}$ commute), Eq. (B11) correctly reproduces the kinetic correction term given in Eq. (A6). Compared to the classical result in Eq. (A6), Eq. (B10) also contains a fourth-order quantum-correction term, which provides a contribution to the pseudopotential and which scales as $\sim q^{2} E_{R}$. The eigenvalues of $H_{\text {eff }}$ yield the Floquet quasienergies. If the eigenstates of $H_{\text {eff }}$ are known, then the Floquet states can be computed up to order $\omega^{-4}$ using the explicit expressions for $F$ derived in Refs. [31,32]. Similarly to the classical analysis above, we find an effective potential up to fourth order in $1 / \omega$, which reads

$$
\begin{aligned}
V_{\mathrm{eff}}(\hat{x}) & =\left[\frac{q}{8} V_{\mathrm{SAW}}+\frac{q^{2}}{32} E_{R}\right] \sin ^{2}(k \hat{x}) \\
& =\varepsilon^{2} E_{S} \sin ^{2}(k \hat{x}),
\end{aligned}
$$

where we introduce the factor

$$
\varepsilon^{2}=\frac{q^{2}}{8}[1+\tilde{q}], \quad \tilde{q}=\frac{E_{R}}{4 E_{S}}=\left(\frac{\hbar k}{2 p_{s}}\right)^{2},
$$

where the momentum $p_{s}$ is given by $p_{s}=m v_{s}$. Within the usual harmonic oscillator (HO) approximation, we obtain the corresponding trapping frequency as

$$
\frac{\omega_{0}}{\omega}=\varepsilon=\frac{q}{2 \sqrt{2}} \sqrt{1+\tilde{q}^{2}} .
$$

\section{APPENDIX C: PHONON-INDUCED COOLING IN THE PRESENCE OF MICROMOTION}

In this appendix, we discuss in detail the phononinduced cooling-heating dynamics and the resulting effective temperature of acoustically trapped charge carriers, with full consideration of the time dependence of the SAWinduced trapping potential. Here, we focus on the relevant decoherence processes due to coupling of the particle's motion to the (thermal) phonon reservoir. Our analysis is built upon the master equation formalism, a tool widely used in quantum optics for studying the irreversible dynamics of a quantum system coupled to a macroscopic environment. We detail the assumptions of our approach and discuss in detail the relevant approximations.

\section{Time-dependent system dynamics}

The system dynamics describing the motion of an electron (of mass $m$ ) exposed to a SAW-induced standing wave is described by the Hamiltonian given in Eq. (3). In the following, we restrict ourselves to the so-called LambDicke regime in which the electron's motion is confined to a region much smaller than the SAW wavelength $\lambda=2 \pi / k$. The corresponding approximation $\cos (k \hat{x}) \approx \mathbb{1}-\left(k^{2} / 2\right) \hat{x}^{2}$ is justified self-consistently below. Dropping the first term $\sim \mathbb{1}$ (which results in an irrelevant, global phase only), the Hamiltonian $H_{S}(t)$ may be written as

$$
H_{S}(t) \approx \frac{\hat{p}^{2}}{2 m}+\frac{m}{2} W(t) \hat{x}^{2},
$$


where $W(t)=-\left(\omega^{2} / 2\right) q \cos (\omega t)$ can be identified as a time-varying spring constant, with the stability parameter $q=V_{\mathrm{SAW}} / E_{S}$. In this form, the Hamiltonian $H_{S}(t)$ and the corresponding dynamics have been studied extensively in the literature (primarily in the context of trapped ions), from both a classical and a quantum-mechanical point of view; see, for example, Refs. [30,33,103]. Still, in order to set up the relevant notation for the subsequent analysis, here we provide a self-contained discussion, closely following Refs. [30,33,103].

Starting out from Eq. (C1), the Heisenberg equations of motion for the electron's position $\hat{x}$ and momentum operators $\hat{p}$ read

$$
\begin{gathered}
\dot{\hat{x}}(t)=\frac{1}{i \hbar}\left[\hat{x}(t), H_{S}(t)\right]=\hat{p}(t) / m, \\
\dot{\hat{p}}(t)=\frac{1}{i \hbar}\left[\hat{p}(t), H_{S}(t)\right]=-m W(t) \hat{x}(t),
\end{gathered}
$$

which, when taken together, yield the well-known quantum Mathieu equation:

$$
\ddot{\hat{x}}(t)+W(t) \hat{x}(t)=0 .
$$

This equation is equivalent to its classical counterpart if one replaces the operator $\hat{x}(t)$ with a function $u(t)$, which satisfies the classical Mathieu equation [30,33,103]. As is well known in the context of trapped ions, stable solutions exist only for certain values of the parameter $q$, which are usually defined in terms of a stability chart; as compared to the standard analysis, here we consider the simplified scenario without any dc voltage [33]. According to Floquet's theorem, such a stable solution $u(t)$ takes on the form

$$
u(t)=\sum_{n=-\infty}^{\infty} c_{2 n} e^{i\left(\omega_{0}+n \omega\right) t}=e^{i \omega_{0} t} \Phi(t),
$$

where $\Phi(t)$ is a periodic function with period $T=2 \pi / \omega$, i.e., $\Phi(t+T)=\Phi(t)$. Following Ref. [30], we consider solutions of the Mathieu equation subject to the boundary conditions

$$
u(0)=1, \quad \dot{u}(0)=i \omega_{0} .
$$

As we show later, this choice of boundary conditions is convenient for the appropriate definition of commutation relations. The (secular) frequency $\omega_{0} / \omega$ is a function of $q$ and the coefficients can be expressed in terms of a continued fraction; see, e.g., Refs. [30,33]. In the limit $q^{2} \ll 1$ it can be shown that $c_{0} \gg\left|c_{ \pm 2}\right|$, such that the solution $u(t)$ is dominated by the so-called secular frequency $\omega_{0} / \omega \approx q /(2 \sqrt{2})$, which is much smaller than the driving frequency $\omega$. In the corresponding pseudopotential regime, a small-amplitude modulation with micromotion frequency $\omega$ is superimposed on the slow (secular) macromotion. To lowest order in $\sim q$, the solution $u(t)$ simplifies to $u(t)=\exp \left[i \omega_{0} t\right]$, without accounting for the micromotion.

Since the solution $u(t)$ and its complex conjugate $u^{*}(t)$ form linearly independent solutions (which are related to each other by the time-inversion symmetry inherent to the Mathieu equation) [30,34], they obey the Wronskian identity

$$
\begin{aligned}
\mathcal{W}(t) & =u^{*}(t) \dot{u}(t)-u(t) \dot{u}^{*}(t) \\
& =u^{*}(0) \dot{u}(0)-u(0) \dot{u}^{*}(0) \\
& =2 i \omega_{0}
\end{aligned}
$$

The second equality simply follows from the fact that $\mathcal{W}(t)$ is a constant of motion. With this normalization, we obtain the sum rule

$$
\sum_{n} c_{n}^{2}\left(\frac{\omega_{0}+n \omega}{\omega_{0}}\right)=1 .
$$

Since $\hat{x}(t)$ and $u(t)$ by definition satisfy the same differential equation, one can construct an operator $\hat{C}(t)$ that consists of an explicitly time-dependent linear combination of the position and momentum operators as

$$
\hat{C}(t)=i \sqrt{\frac{m}{2 \hbar \omega_{0}}}[u(t) \dot{\hat{x}}(t)-\dot{u}(t) \hat{x}(t)],
$$

but which (being proportional to the Wronskian $\mathcal{W}$ ) turns out to be a constant of motion $[30,33,103]$. Then, since

$$
\hat{C}(t)=\hat{C}(0)=\frac{1}{\sqrt{2 m \hbar \omega_{0}}}\left[m \omega_{0} \hat{x}(0)+i \hat{p}(0)\right],
$$

one can readily identify $\hat{C}(t)$ with the well-known annihilation operator associated with a static harmonic oscillator of mass $m$ and frequency $\omega_{0}$ as

$$
\hat{C}(t)=\hat{C}(0)=A,
$$

with the usual standard commutation relation

$$
\left[A, A^{\dagger}\right]=1 .
$$

This static potential harmonic oscillator is usually referred to as a reference oscillator [30]. Since the operator $A$ is time independent, the same is true for

$$
N=A^{\dagger} A,
$$


whose eigenstates are simply the familiar Fock states of the (static potential) reference oscillator, with the standard ladder algebra

$$
\begin{gathered}
A|n\rangle_{\omega_{0}}=\sqrt{n}|n-1\rangle_{\omega_{0}}, \\
A^{\dagger}|n\rangle_{\omega_{0}}=\sqrt{n+1}|n+1\rangle_{\omega_{0}},
\end{gathered}
$$

yielding directly $N|n\rangle=n|n\rangle_{\omega_{0}}$.

The Heisenberg operators $\hat{x}(t)$ and $\hat{p}(t)$ can then be expressed in terms of the classical Mathieu solutions $u(t)$ as well as the (time-independent) creation and annihilation operators of the reference oscillator as

$$
\begin{gathered}
\hat{x}(t)=\sqrt{\frac{\hbar}{2 m \omega_{0}}}\left[u^{*}(t) A+u(t) A^{\dagger}\right], \\
\hat{p}(t)=\sqrt{\frac{\hbar m}{2 \omega_{0}}}\left[\dot{u}^{*}(t) A+\dot{u}(t) A^{\dagger}\right] .
\end{gathered}
$$

Accordingly, the time dependence of the Heisenberg operators $\hat{x}(t)$ and $\hat{p}(t)$ is captured entirely by the classical Mathieu equation $u(t)$ and its complex conjugate. Note that $[\hat{x}(t), \hat{p}(t)]=\frac{\hbar}{2 \omega_{0}} \mathcal{W}(t)=i \hbar$, as desired. For later reference, here we also define the Heisenberg operator for the kinetic energy as

$$
\begin{aligned}
\frac{\hat{p}^{2}(t)}{2 m}= & \frac{\hbar}{4 \omega_{0}}\left[|\dot{u}(t)|^{2}\left(A^{\dagger} A+A A^{\dagger}\right)\right. \\
& \left.+\left(\dot{u}^{*}(t)\right)^{2} A^{2}+(\dot{u}(t))^{2}\left(A^{\dagger}\right)^{2}\right] .
\end{aligned}
$$

Since the annihilation (creation) operators $A\left(A^{\dagger}\right)$ associated with the reference oscillator satisfy the usual algebra, in complete analogy to the standard oscillator one may define a set of basis states (in the Schrödinger picture) labeled as $|n ; t\rangle$, with $n=0,1,2, \ldots$, which form the dynamic counterpart of the harmonic oscillator Fock states. The states $|n ; t\rangle$ are not stationary states, but do depend explicitly on time, as indicated by the argument $t$ in the ket vector [103]. The ground state of the reference oscillator $|n=0\rangle_{\omega_{0}}$ obeys the condition

$$
A|n=0\rangle_{\omega_{0}}=\hat{C}(t)|n=0\rangle_{\omega_{0}}=0 .
$$

We can relate the Heisenberg operator $\hat{C}(t)$ to its counterpart in the Schrödinger picture $\hat{C}_{S}(t)$ as $\hat{C}_{S}(t)=$ $U(t) \hat{C}(t) U^{\dagger}(t)$, with the unitary operator $U(t)$, which fulfills

$$
\dot{U}(t)=-i H_{S}(t) U(t) .
$$

Explicitly, we find

$$
\hat{C}_{S}(t)=\frac{1}{2 i}\left[\dot{u}(t) \sqrt{\frac{2 m}{\hbar \omega_{0}}} \hat{x}-u(t) \sqrt{\frac{2}{m \hbar \omega_{0}}} \hat{p}\right] .
$$

Then, Eq. (C21) can be rewritten as

$$
\hat{C}_{S}(t) U(t)|n=0\rangle_{\omega_{0}}=\hat{C}_{S}(t)|n=0 ; t\rangle=0,
$$

where we introduce the state $|n=0 ; t\rangle$ in the Schrödinger picture which evolves unitarily starting from the ground state of the reference oscillator as $|n=0 ; t\rangle=U(t)|n=0\rangle_{\omega_{0}}$. The ladder-operator relations stated in Eqs. (C16) and (C17) for the reference oscillator can easily be transferred to the Schrödinger picture, yielding

$$
\begin{aligned}
\hat{C}_{S}(t)|n ; t\rangle & =\sqrt{n}|n-1 ; t\rangle, \\
\hat{C}_{S}^{\dagger}(t)|n ; t\rangle & =\sqrt{n+1}|n+1 ; t\rangle,
\end{aligned}
$$

implying $N_{S}(t)|n ; t\rangle=n|n ; t\rangle$, with $N_{S}(t)=\hat{C}_{S}^{\dagger}(t) \hat{C}_{S}(t)$. Since the Schrödinger operators $\hat{C}_{S}(t), \hat{C}_{S}^{\dagger}(t)$ act as shift operators for the Floquet states $|n ; t\rangle$, they will be referred to as Floquet shift operators [34]. Therefore, all other states forming the complete orthonormal basis $\{|n ; t\rangle\}$ can be constructed by repeated operation on the ground state with the Schrödinger creation operator $\hat{C}_{S}(t)$ (with the proper normalization) as

$$
|n ; t\rangle=\frac{\left[\hat{C}_{S}(t)\right]^{n}}{\sqrt{n !}}|n=0 ; t\rangle .
$$

When expressing this equation in coordinate space, the micromotion appears in the wave functions as a pulsation with the period $T=2 \pi / \omega$ [30]. Although the states $|n ; t\rangle$ are not energy eigenstates (since they periodically exchange energy with the driving field), they are typically referred to as quasistationary states, because for stroboscopic times (that are integer multiples of the driving period $T$ ) the full evolution $U(t)$ boils down to multiplying the wave function by a simple phase factor (as is the case for standard stationary states for all times). Because of the periodicity of the micromotion, the quantum number $n$ (labeling the quasienergy states) can thus be tied to the electron's energy averaged over a period $T=2 \pi / \omega$ of the drive frequency. This connection is explored in greater detail below.

\section{System-bath model}

While our previous discussion has exclusively focused on the time-dependent system's dynamics [as described by the Hamiltonian $H_{S}(t)$ given in Eq. (C1)], in the following we develop a microscopic dissipative model, which describes the electron's motional coupling to the (thermal) phonon reservoir. 
The global Hamiltonian, describing both the electronic motion as well as the phonon reservoir, can be formally decomposed as

$$
H(t)=H_{S}(t)+H_{B}+H_{I} .
$$

Here, the time-dependent system Hamiltonian $H_{S}(t)$ is given in Eq. (C1). The Hamiltonian for the phonon bath $H_{B}$ is of the usual form:

$$
H_{B}=\sum_{\mathbf{q}, s} \omega_{\mathbf{q}, s} a_{\mathbf{q}, s}^{\dagger} a_{\mathbf{q}, s},
$$

where $a_{\mathbf{q}, s}^{\dagger}\left(a_{\mathbf{q}, s}\right)$ creates (annihilates) an acoustic phonon with wave vector $\mathbf{q}=\left(\mathbf{q}_{\|}, q_{z}\right)$, with polarization $s$ and dispersion $\omega_{\mathbf{q}, s}$. Optical phonons can be disregarded at sufficiently low energies as considered here [104]. Following Ref. [40], generically the electron-phonon interaction takes on the form

$$
H_{I}=\sum_{\mathbf{q}, s} W_{\mathbf{q}, s} a_{\mathbf{q}, s} e^{i \mathbf{q} \cdot \hat{\mathbf{r}}}+\text { H.c. },
$$

with $\hat{\mathbf{r}}=(\hat{x}, \hat{y}, \hat{z})$ denoting the electron's three-dimensional position operator. The coupling constant $W_{\mathbf{q}, s}$ comprises both the deformation potential as well as the piezoelectric coupling mechanism [40,104]; it strongly depends on specific material properties, but can be left unspecified for the sake of our discussion. For low-dimensional quasi2D systems as we consider here, the Hamiltonian $H_{I}$ may be simplified by projecting the electronic motional degrees of freedom onto the lowest electronic orbital $\psi_{0}(z)$, leading to

$$
H_{I} \approx \sum_{\mathbf{q}, s} \mathcal{F}\left(q_{z}\right) W_{\mathbf{q}, s} a_{\mathbf{q}, s} e^{i \mathbf{q}_{\|} \cdot \hat{\mathbf{r}}_{\|}}+\text {H.c. }
$$

with the in-plane position operator $\hat{\mathbf{r}}_{\|}=(\hat{x}, \hat{y})$. The form factor $\mathcal{F}\left(q_{z}\right)=\int d z e^{i q_{z} z}\left|\psi_{0}(z)\right|^{2}$ introduces a momentum cutoff, with $\mathcal{F}\left(q_{z}\right)$ approaching unity in the limit $\left|q_{z}\right| \ll$ $d^{-1}$ and vanishing for $\left|q_{z}\right| \gg d^{-1}$; here, $d \sim 10 \mathrm{~nm}$ denotes the size of the quantum well along the $z$ axis [104]. For the sake of clarity, here we consider a quasi-one-dimensional structure (a quantum wire) where the electron's motion is restricted to the $x$ direction; compare our previous discussion in Sec. C1. In this case, the electron-phonon interaction reduces to

$$
H_{I} \approx \sum_{\mathbf{q}, s} \tilde{W}_{\mathbf{q}, s} a_{\mathbf{q}, s} e^{i q \hat{x}}+\text { H.c. }
$$

where the coupling $\tilde{W}_{\mathbf{q}, s}$ accounts for transversal confinement in both the $y$ and $z$ direction; moreover, we set $q=q_{x}$. Along the lines of Sec. C 1, again we restrict ourselves to the Lamb-Dicke regime in which the electron's motion is confined to a region much smaller than the wavelength of the relevant, resonant phonon modes. Then, taking $e^{i q \hat{x}} \approx \mathbb{1}+i q \hat{x}$, and introducing displaced bosonic bath modes as

$$
\begin{gathered}
b_{\mathbf{q}, s}=-i\left(a_{\mathbf{q}, s}+\tilde{W}_{\mathbf{q}, s}^{*} / \omega_{\mathbf{q}, s}\right), \\
b_{\mathbf{q}, s}^{\dagger}=i\left(a_{\mathbf{q}, s}^{\dagger}+\tilde{W}_{\mathbf{q}, s} / \omega_{\mathbf{q}, s}\right),
\end{gathered}
$$

we finally arrive at the following microscopic system-bath model with bilinear coupling between the system [as described by Eq. (C1)] and a bath of noninteracting harmonic oscillators (i.e., the phonon reservoir):

$$
\begin{gathered}
H_{B}=\sum_{\nu} \omega_{\nu} b_{\nu}^{\dagger} b_{\nu}, \\
H_{I}=\hat{x} \sum_{\nu} g_{\nu} \hat{x}_{\nu}+\hat{x}^{2} \sum_{\nu} \frac{g_{\nu}^{2}}{2 m_{\nu} \omega_{\nu}^{2}} .
\end{gathered}
$$

where, to simplify the notation, we introduce the multiindex $\nu=(\mathbf{q}, s)$ and $g_{\nu}$ specifies the coupling strength between the system and each bath oscillator mode $\nu$. Following the standard procedure in the literature, in Eq. (C35) we also include a correction term that acts in the Hilbert space of the particle only and compensates for a renormalization of the potential $V(\hat{x}, t)=(m / 2) W(t) \hat{x}^{2}$ stemming from the system-reservoir coupling [34,105]. In this model, the reservoir spectral density, defined as

$$
J(\omega)=\pi \sum_{\nu} \frac{g_{\nu}^{2}}{2 m_{\nu} \omega_{\nu}} \delta\left(\omega-\omega_{\nu}\right),
$$

encodes all features of the environment relevant for the reduced system description [34].

\section{Quantum master equation, quasistationary state, and effective temperature}

The time-dependent, dissipative quantum system described by Eqs. (C27), (C1), (C34), and (C35), commonly referred to as a parametrically driven, dissipative harmonic quantum oscillator, has been studied in great detail previously in Ref. [34]. Within one unified BornMarkov and Floquet framework, the authors of Ref. [34] have derived a quantum master equation for the electronic motion, fully taking into account the explicit time dependence of the system Hamiltonian $H_{S}(t)$.

Master equation.-By tracing out the unobserved degrees of freedom of the phonon reservoir, Kohler et al. derive an effective equation of motion for the reduced, electronic density matrix $\rho$, which is irreversibly coupled to a thermal phonon reservoir [34]. In addition to the standard assumptions of a weak system-reservoir coupling (Born approximation), and a short reservoir correlation time 
(Markov approximation), the analysis has been restricted to an Ohmic spectral density where $J(\omega) \sim \omega$ (which, however, may be generalized to a more general setting straightforwardly). Under these conditions, the central master equation can be written as

$$
\dot{\rho}=-\frac{i}{\hbar}\left[\hat{H}_{S}(t), \rho\right]+\mathcal{L}_{\gamma} \rho,
$$

with

$$
\mathcal{L}_{\gamma} \rho=\gamma(N+1) \mathcal{D}\left[\hat{C}_{S}(t)\right] \rho+\gamma N \mathcal{D}\left[\hat{C}_{S}^{\dagger}(t)\right] \rho .
$$

Here, $\mathcal{D}[c] \rho=c \rho c^{\dagger}-\frac{1}{2}\left\{c^{\dagger} c, \rho\right\}$ is a dissipator of Lindblad form, $\gamma$ denotes the effective, incoherent damping rate due to coupling to the thermal phonon reservoir, and

$$
N=\sum_{n} c_{2 n}^{2} \frac{\omega_{0}+n \omega}{\omega_{0}} \bar{n}_{\mathrm{th}}\left(\omega_{0}+n \omega\right),
$$

with $\bar{n}_{\text {th }}(\omega)=\left(\exp \left[\hbar \omega / k_{B} T\right]-1\right)^{-1}$, refers to a generalized effective thermal-bath occupation number. Note that Eq. (C37) retains the periodicity of the driving and exhibits Lindblad form. Moreover, the dissipative part of Eq. (C37) is of the same form as for the well-known undriven dissipative harmonic oscillator, with the Floquet shift operators defined in Eqs. (C23) and (C25) replacing the usual creation and annihilation operators. Note that in the pseudopotential limit (where $c_{0}$ is much larger than all other Floquet coefficients), the effective thermal occupation reduces to $N=\bar{n}_{\text {th }}\left(\omega_{0}\right)$, which is the standard bosonic thermal occupation at the secular frequency $\omega_{0}$.

The master equation given in Eq. (C37) is valid provided that the following conditions are satisfied [34]. (i) First, the Markov approximation is satisfied provided that autocorrelations of the bath (which typically decay on a time scale $\sim \hbar / k_{B} T$ ) decay quasi-instantaneously on the time scale of system correlations $\sim \gamma^{-1}$. In principle, the damping rate $\gamma$ should be replaced by the thermally enhanced rate $\gamma_{\text {eff }}=\gamma(N+1)$; however, we are interested mostly in the low-temperature, pseudopotential regime where $\gamma_{\text {eff }} \approx \gamma$. Thus, the Markov approximation yields the condition $\hbar \gamma \ll k_{B} T$. (ii) Second, the (weak-coupling) Born approximation holds provided that the dissipative damping rate $\gamma$ is small compared to the relevant system's transition frequencies, yielding the requirement $\gamma \ll \omega_{0}$. Taking together conditions (i) and (ii) (and setting $\hbar=1$ for the moment) gives the requirement

$$
\gamma \ll \omega_{0}, k_{B} T,
$$

which (as we show below) comprises the regime for ground-state cooling where $\gamma \ll k_{B} T \ll \omega_{0}$. (iii) Finally, when deriving Eq. (C37), the reservoir spectral density $J(\omega)$ is assumed to be Ohmic [i.e., $J(\omega) \sim \omega$ ].
Quasistationary state.-Using Eq. (C25), the (asymptotic) quasistationary solution $\rho_{\mathrm{ss}}(t)$ associated with the master equation (C37) is readily found to be

$$
\rho_{\mathrm{ss}}(t)=\frac{1}{N+1} \sum_{n=0}^{\infty}\left(\frac{N}{N+1}\right)^{n}|n ; t\rangle\langle n ; t|,
$$

where $|n ; t\rangle$ refer to the generalized (time-dependent) Fock states as discussed above [34]. The quasistationary solution $\rho_{\mathrm{ss}}(t)$ is dark with respect to the phonon-induced dissipation, that is, $\mathcal{L}_{\gamma} \rho_{\mathrm{ss}}(t)=0$ for all times, and, being a mixture of the Floquet solutions $|n ; t\rangle$, evolves periodically with the period of the driving field, i.e., $\rho_{\mathrm{ss}}(t+T)=\rho_{\mathrm{ss}}(t)$.

While the notion of temperature becomes ambiguous for an explicitly time-dependent problem as considered here, in the following we adopt the reasoning presented in Ref. [33] and take the mean kinetic energy [defined as the quantum kinetic energy $\overline{\left\langle\hat{p}^{2}(t)\right\rangle} / 2 m$, time averaged over one period $T=2 \pi / \omega$ of the fast micromotion] as our figure of merit for assessing the cooling-heating dynamics in more detail. To do so, let us first transform our analysis into a frame that is moving with the electron. Formally, this transformation is defined as $Q=U^{\dagger}(t) \rho U(t)$, with the unitary operator that satisfies

$$
\dot{U}(t)=-i H_{S}(t) U(t) .
$$

Then, in the corresponding interaction picture (which coincides with the Heisenberg picture defined in Sec. C 1) the dynamics described by Eq. (C37) reduces to a purely dissipative master equation, $\dot{Q}=\mathcal{L} Q$,

$$
\dot{\varrho}=\gamma(N+1) \mathcal{D}[A] \varrho+\gamma N \mathcal{D}\left[A^{\dagger}\right] \varrho,
$$

where $A\left(A^{\dagger}\right)$ refers to the time-independent annihilation (creation) operator associated with the reference oscillator discussed in Sec. C1. Since the Liouvillian $\mathcal{L}$ is time independent, one can easily explain the phononinduced cooling dynamics via the eigenstates of $A^{\dagger} A$, as defined in Sec. C 1. For simplicity, let us focus on the pseudopotential regime where $N \approx \bar{n}_{\text {th }}\left(\omega_{0}\right)$, as discussed above; then, for sufficiently low temperatures $\left(k_{B} T \ll\right.$ $\left.\hbar \omega_{0}\right)$ the cooling dynamics dominate over the heating processes such that, at the end of the cooling process, we have $\left\langle A^{\dagger} A\right\rangle=\left\langle A^{2}\right\rangle=\left\langle A^{\dagger} A^{\dagger}\right\rangle=0$. In this regime, the expectation value for the quantum kinetic energy reduces to

$$
\frac{\left\langle\hat{p}^{2}(t)\right\rangle}{2 m} \stackrel{\text { cooling }}{\longrightarrow} \frac{\hbar}{4 \omega_{0}}|\dot{u}(t)|^{2}
$$

as one can readily deduce from Eq. (C20). Averaging this expression (which still fully accounts for the time 
dependence of the potential) over one micromotion period, we obtain

$$
\begin{aligned}
\frac{\overline{\left\langle\hat{p}^{2}(t)\right\rangle}}{2 m} & =\frac{\hbar}{4 \omega_{0}} \sum_{n}\left|c_{2 n}\right|^{2}\left(\omega_{0}+n \omega\right)^{2} \\
& =\frac{\hbar \omega_{0}}{4}+\Delta_{\text {heat }},
\end{aligned}
$$

where we separate the residual kinetic zero-point motion in the ground state of the secular reference oscillator $\sim \hbar \omega_{0} / 4$ from the nonzero heating term,

$$
\Delta_{\text {heat }}=\frac{\hbar}{4 \omega_{0}} \sum_{n \neq 0}\left|c_{2 n}\right|^{2}\left(\omega_{0}+n \omega\right)^{2},
$$

which may be viewed as micromotion-induced heating. While the full expression given in Eq. (C45) can be evaluated numerically using the well-known solutions of the Mathieu equation (compare, for example, Ref. [33]), a simple estimate (for $q^{2} \ll 1$ ) shows $\Delta_{\text {heat }} \gtrsim \hbar \omega_{0} / 4$. Therefore, in agreement with the results presented in Ref. [33] for trapped ions, we then find $\overline{\left\langle\hat{p}^{2}(t)\right\rangle} / 2 m \gtrsim$ $\hbar \omega_{0} / 2$ for the time-averaged kinetic energy in the pseudopotential regime, which coincides with twice the residual kinetic zero-point motion in the ground state of the reference oscillator.

In conclusion, our analysis shows that micromotion does lead to some heating as compared to the naive estimate based on the slow secular motion only, but (in the pseudopotential regime of interest, where $q^{2} \ll 1$ ) this apparent heating mechanism is strongly suppressed and amounts to merely a factor of 2 increase only in the particle's time-averaged kinetic energy.

\section{Exact numerical simulations and discussion}

Setup.-Since the electronic dynamics described by Eq. (C37) are purely Gaussian, an exact solution is feasible. Therefore, in the following we complement our analytical findings with numerically exact simulations for the electron's dynamics. Based on Eq. (C37), one can readily derive a closed dynamical equation,

$$
\frac{d}{d t} \mathbf{v}=\mathcal{M}(t) \mathbf{v}+\mathbf{C}(t),
$$

where $\mathbf{v}$ is a five-component vector comprising the firstand second-order moments; that is, $\mathbf{v}=\left(\langle\hat{x}\rangle_{t},\langle\hat{p}\rangle_{t},\left\langle\hat{x}^{2}\right\rangle_{t}\right.$, $\left.\left\langle\hat{p}^{2}\right\rangle_{t},\langle\hat{x} \hat{p}+\hat{p} \hat{x}\rangle_{t}\right)^{\top}$. Since the first- and second-order moments are decoupled, the dynamical matrix $\mathcal{M}$ is of block-diagonal form.

Numerical results.-As illustrated in Fig. 3, in the regime $q^{2} \ll 1$ we numerically find that (i) the electronic motion can be described very well by a simple damped harmonic oscillator with secular frequency $\omega_{0}$, (ii) the electronic motion is cooled by the phonon reservoir, and (iii) the Lamb-Dicke approximation is well satisfied. Let us elaborate on these statements in some more detail. (i) When disregarding micromotion, the dynamics can approximately be described by a simple damped harmonic oscillator with secular frequency $\omega_{0}$. As shown in Fig. 3, the effective, time-independent master equation,

$$
\begin{aligned}
\dot{\rho}= & -i \omega_{0}\left[a^{\dagger} a, \rho\right]+\gamma\left(\bar{n}_{\mathrm{th}}\left(\omega_{0}\right)+1\right) \mathcal{D}[a] \rho \\
& +\gamma \bar{n}_{\mathrm{th}}\left(\omega_{0}\right) \mathcal{D}\left[a^{\dagger}\right] \rho,
\end{aligned}
$$

with $a\left(a^{\dagger}\right)$ denoting the usual annihilation (creation) operators for the canonical harmonic oscillator, captures well the most pertinent features of the electronic dynamics, provided that $q^{2} \ll 1$; compare the dashed orange line in Fig. 3. (ii) As suggested by our analytical analysis, the phonon reservoir provides an efficient cooling mechanism for the electron provided that the host temperature is sufficiently low; that is, $k_{B} T \ll \omega_{0}$. (iii) Regarding the last statement (iii), we numerically verify that both the expectation value for the electron's motion as well as the corresponding fluctuations are small compared to the SAW wavelength, $\lambda=2 \pi / k$, i.e., $k\langle\hat{x}\rangle_{t} \ll 1$ and $k \sigma_{x} \ll 1$, with $\sigma_{x}^{2}=\left\langle\hat{x}^{2}\right\rangle_{t}-\langle\hat{x}\rangle_{t}^{2}$. Furthermore, the Lamb-Dicke approximation underlying the bilinear system-bath interaction Hamiltonian [compare Eq. (C35)] can be justified as follows. Since the effective transition frequency $\omega_{0}$ is much smaller than the SAW driving frequency $\left(\omega_{0}=\varepsilon \omega\right.$, with $\varepsilon \ll 1)$, the same is true for the relevant phonon wave number $k_{0}$. Using the relation $\omega_{0}=v_{s}^{(b)} k_{0}$ (where $v_{s}^{(b)}$ refers to the speed of sound associated with some relevant bulk phonon mode), the latter can be expressed as $k_{0}=$ $\varepsilon\left(v_{s} / v_{s}^{(b)}\right) k$ (with $v_{s}$ denoting the speed of sound of the SAW mode driven by the IDTs, as usual). Therefore, even for higher Rayleigh SAW modes whose speed of sound $v_{s}$ may exceed the lowest value of $v_{s}^{(b)}$, our approximate treatment of the system-bath Hamiltonian is well justified, provided that $v_{s} \lesssim v_{s}^{(b)} / \varepsilon$ holds. Note that materialengineering strategies as discussed in the main text would increase $v_{s}$ in the same way as $v_{s}^{(b)}$, providing a very good justification for our linearized Hamiltonian Eq. (C35) since $k_{0} \lesssim \varepsilon k$.

Finally, the parameter regime of interest is summarized and discussed extensively in Sec. II of the main text, while the experimental feasibility thereof is discussed in Sec. III.

\section{APPENDIX D: CASE STUDY AND PRACTICAL CONSIDERATIONS}

In this appendix, we provide further details regarding several practical considerations that are relevant for a faithful experimental realization of our proposal. First, 
TABLE II. Important (energy) scales for an exemplary setup with $E_{S}=1 \mathrm{meV}$ and $f=50 \mathrm{GHz}$. $d$ denotes the distance between the screening layer and the 2DEG.

\begin{tabular}{lccccccccc}
\hline \hline$\hbar \omega[\mu \mathrm{eV}]$ & $q=V_{\mathrm{SAW}} / E_{S}$ & $\hbar \omega_{0}[\mu \mathrm{eV}]$ & $V_{0}[\mu \mathrm{eV}]$ & $n_{b}=V_{0} / \hbar \omega_{0}$ & $a=\lambda / 2[\mathrm{~nm}]$ & $d[\mathrm{~nm}]$ & $t[\mu \mathrm{eV}]$ & $U[\mu \mathrm{eV}]$ & $k_{B} T[\mu \mathrm{eV}]$ \\
\hline 207 & $0.5-0.7$ & $37-51$ & $31-61$ & $0.85-1.2$ & 180 & $10-100$ & $0.7-1.8$ & $5-270$ & $1-10$ \\
\hline \hline
\end{tabular}

we provide a comprehensive overview of the key quantities of our analysis and self-consistent estimates thereof. Next, we address microwave-induced heating effects. Lastly, we discuss electron spin decoherence effects due to (nuclear) spin noise.

Case study.-Typical parameter regimes for the key quantities of our analysis are given in Table II. The parameters are chosen self-consistently with respect to the requirements derived in the main text; see Eq. (6). Note that the high-SAW frequencies lead to large energy scales in the effective (harmonic-oscillator) problem. For comparison, ions are typically confined in traps with harmonic-oscillator energy $\hbar \omega_{0} \sim 10 \mathrm{MHz}$ [30]. For the SAW velocity $v_{s}$, we assume an ultrafast PSAW mode in AlN/diamond $\left(v_{s} \approx 18 \mathrm{~km} / \mathrm{s}\right)$, as described in the main text, and a corresponding effective hole mass $m=1.1 m_{0}$ in the host material GaN where the 2DEG is located.

Heating.-In order to avoid excessive heating of the effective electron temperature above the dilution-fridge temperature in the presence of $\mathrm{rf}$ driving, we either need (i) the heat dissipation $W_{\text {heat }}$ to be balanced by the applied cooling power $P_{\text {cool }}$ (for which, in an actual experiment, the way the sample is heat sunk is very important) or (ii) the heat dissipation to be too slow to change the electron's temperature on relevant experimental time scales after the IDT-induced driving has been turned on. In the following, we argue why the requirements (i) and (ii) can both be fulfilled under realistic conditions. (i) First, recall that our proposal is based on low-power SAWs (as a direct consequence of the limitations imposed by Mathieu's equation) [60]. Since the potential amplitude due to a single IDT is limited by Mathieu-like stability arguments as $V_{\mathrm{IDT}}=V_{\mathrm{SAW}} / 2=(q / 2) E_{S} \lesssim 0.5 \mathrm{meV}$, the proposed setup operates at $\mathrm{SAW}$-induced amplitudes that are about 2 orders of magnitude smaller than what is common for SAW-induced electron transport experiments (where typically $\left.V_{\mathrm{IDT}} \approx 40 \mathrm{meV}[13,61]\right)$. Based on experimental results presented in Refs. [13,106,107], we find that SAW amplitudes $V_{\mathrm{IDT}} \approx 1 \mathrm{meV}$ can be reached with an applied rf power $P \approx-10 \mathrm{dBm}(0.1 \mathrm{~mW})$, in the desired SAW frequency range $\omega / 2 \pi \approx 30 \mathrm{GHz}$ (as needed to enter the pseudopotential regime), whereas high-amplitude electron transport measurements operate at $P>$ $+10 \mathrm{dBm}(10 \mathrm{~mW})$ [13]. This estimate is based on experiments with relatively wide IDTs in GaAs; therefore, the power budget $P$ could be further reduced (if needed) by reducing the width $W$ of the IDTs (which is typically several hundreds of $\mu \mathrm{m}$ long [108], i.e., much longer than necessarily required for an acoustic trap or lattice) and/or using strongly piezoelectric materials $[19,20,109]$ where the electromechanical coupling efficiency is much larger than for the weakly piezoelectric material GaAs. Heating effects as a function of the applied rf power $P$ have been investigated experimentally in detail in Refs. [106,107]: Here, at a comparatively large microwave power $P=$ $+5 \mathrm{dBm}$, the SAW-induced heating has been measured to be $W_{\text {heat }}^{\mathrm{SAW}} \approx 0.1 \mathrm{~mW}$. We may estimate this source of heating as $W_{\text {heat }}^{\mathrm{SAW}} \approx \hbar \omega \times\left(V_{\mathrm{SAW}} / V_{0}\right)^{2} \kappa$, where $\hbar \omega$ is the energy of a single phonon and the second factor gives the total phonon loss rate in terms of the phonon number $N_{\mathrm{ph}} \approx\left(V_{\mathrm{SAW}} / V_{0}\right)^{2}$ and the decay rate $\kappa=\omega / Q$; here, $V_{0}$ refers to the amplitude associated with a single phonon [98] and $Q$ is the quality factor associated with the driven SAW mode. However, it has been shown in Ref. [107] that $W_{\text {heat }}^{\text {SAW }}$ accounts for $\sim 10 \%$ of the overall heating only, due to the limited efficiency of the IDTs. While this ratio may be improved with more sophisticated IDT designs $[19,20,110]$, an overall heating of $W_{\text {heat }} \approx 10 W_{\text {heat }}^{\text {SAW }} \approx$ $1 \mathrm{~mW}$ is still compatible with the cooling power of state-of-the-art dilution refrigerators, which can reach $P_{\text {cool }}=1 \mathrm{~mW}$ at $T \approx 100 \mathrm{mK}$ [111]; here, to maximize the cooling efficiency in an actual experiment, attention should be paid to the specific way the sample is heat sunk. Since the proposed AL setup operates at much lower rf power levels $[P \lesssim-10 \mathrm{dBm}(0.1 \mathrm{~mW})$ as compared to $P=+5 \mathrm{dBm}(3 \mathrm{~mW})]$, the overall heat dissipation $W_{\text {heat }}$ can be balanced by the applied cooling power $P_{\text {cool }}$ for the specific parameters under consideration. This finding is further supported by the experiments presented in Refs. [106,107], where for low-power SAWs no significant heating above the base temperature has been observed. (ii) Second, the IDTs generating the SAWs can be placed very far away from the center of the trap, without losing acoustic power, thereby reducing local heat dissipation near the center of the trap due to the applied rf power. For example, in Ref. [13] (and many similar setups) the SAW transducer has been placed approximately $2 \mathrm{~mm}$ away from the center of the sample. In this way, the dominant local heating at the IDT may be suppressed efficiently, at least on time scales that are short compared to the one set by the material-specific thermal diffusivity (which specifies the rate of transfer of heat from the IDT to the cold center of the trap). While this time scale is strongly material dependent, a rough estimate for GaAs shows that it can lie in the millisecond range (for IDTs placed $\sim 1 \mathrm{~mm}$ away from the center of the trap), which is much longer than any relevant experimental time scale. This reasoning is also in 
line with experimental results showing that the effective temperature increase could be further reduced when using pulsed schemes rather than continous wave (CW) [106]; note that this approach is fully compatible with our discussion on optimized driving schemes. In summary, we conclude that for realistic cooling powers and/or IDTs placed sufficiently far away from the center of the trap, microwave-induced heating effects should not lead to a significant increase of the effective particle temperature (as compared to the base temperature) since the AL setup is based on low-amplitude SAWs with $V_{\text {IDT }} \lesssim 0.5 \mathrm{meV}$, as a direct consequence of the Mathieu-type stability arguments.

Nuclear spin noise.- The observation of coherent spin physics as outlined in Sec. IV may be impeded by electron spin decoherence. For GaAs-based systems, the electron spin coherence time scale will be largely limited by the relatively strong hyperfine interaction between the electronic spin and the nuclei in the host environment [112], resulting in a random, slowly evolving magnetic (Overhauser) field for the electronic spin, and eventually leading to a loss of spin coherence on a time scale $\sim T_{2}^{\star}$. The latter depends on the number of nuclear spins the electron effectively interacts with. Since the electron's spatial extension $\Delta x / a \approx$ $1 /\left(\pi \sqrt{2 n_{b}}\right)$ is comparable to the typical size of gate-defined quantum dots for realistic parameter values, we estimate $T_{2}^{\star} \sim 15 \mathrm{~ns}$ [112]. Then, in the first approximation, the detrimental effects due to Overhauser noise may be neglected provided that the condition $J \gg 1 / T_{2}^{\star}$ is fulfilled, i.e., if coherent spin exchange $\sim 1 / J$ is much faster than electron spin dephasing. According to our estimates provided above, this regime is within reach even for GaAs-based systems, where electron spin dephasing is known to be relatively fast [112]. In this respect, even more promising estimates apply to nuclear-spin-free systems, such as ${ }^{28} \mathrm{Si} / \mathrm{SiGe}$, where the influence of nuclear spins on the electron spins is largely eliminated [84]. While such a silicon-based setup will require a more sophisticated heterostructure including some piezoelectric layer on top (as has been studied experimentally in Ref. [24]), it should profit from significantly prolonged dephasing times $T_{2}^{\star}>100 \mu \mathrm{s}$ [113]. Finally, as argued, for example, in Ref. [14], Overhauser-field-induced spin dephasing can be suppressed based on motional-narrowing techniques, when moving around the acoustic dots (lattice sites) such that the electron effectively samples many different Overhauser fields.

[1] A. Ashkin, History of Optical Trapping and Manipulation of Small-Neutral Particle, Atoms, and Molecules, IEEE J. Sel. Top. Quantum Electron. 6, 841 (2000).

[2] M. J. Lang and S. M. Block, Laser-Based Optical Tweezers, Am. J. Phys. 71, 201 (2003).

[3] M. H. Anderson, J. R. Ensher, M. R. Matthews, C. E. Wieman, and E. A. Cornell, Observation of Bose-Einstein
Condensation in a Dilute Atomic Vapor, Science 269, 198 (1995).

[4] C. C. Bradley, C. A. Sackett, J. J. Tollett, and R. G. Hulet, Evidence of Bose-Einstein Condensation in an Atomic Gas with Attractive Interactions, Phys. Rev. Lett. 75, 1687 (1995).

[5] K. B. Davis, M.-O. Mewes, M. R. Andrews, N. J. van Druten, D. S. Durfee, D. M. Kurn, and W. Ketterle, BoseEinstein Condensation in a Gas of Sodium Atoms, Phys. Rev. Lett. 75, 3969 (1995).

[6] R. Blatt and C.F. Roos, Quantum Simulations with Trapped Ions, Nat. Phys. 8, 277 (2012).

[7] I. Bloch, J. Dalibard, and W. Zwerger, Many-Body Physics with Ultracold Gases, Rev. Mod. Phys. 80, 885 (2008).

[8] I. Bloch, J. Dalibard, and S. Nascimbène, Quantum Simulations with Ultracold Quantum Gases, Nat. Phys. 8, 267 (2012).

[9] R. Hanson, L. P. Kouwenhoven, J. R. Petta, S. Tarucha, and L. M. K. Vandersypen, Spins in Few-Electron Quantum Dots, Rev. Mod. Phys. 79, 1217 (2007).

[10] M. J. A. Schuetz, M. G. Moore, and C. Piermarocchi, Trionic Optical Potential for Electrons in Semiconductors, Nat. Phys. 6, 919 (2010).

[11] J. Cunningham, V. I. Talyanskii, J. M. Shilton, M. Pepper, M. Y. Simmons, and D. A. Ritchie, Single-Electron Acoustic Charge Transport by Two Counterpropagating Surface Acoustic Wave Beams, Phys. Rev. B 60, 4850 (1999).

[12] J. A. H. Stotz, R. Hey, P. V. Santos, and K. H. Ploog, Coherent Spin Transport through Dynamic Quantum Dots, Nat. Mater. 4, 585 (2005).

[13] S. Hermelin, S. Takada, M. Yamamoto, S. Tarucha, A. D. Wieck, L. Saminadayar, C. Bäuerle, and T. Meunier, Electrons Surfing on a Sound Wave as a Platform for Quantum Optics with Flying Electrons, Nature (London) 477, 435 (2011).

[14] R. P. G. McNeil, M. Kataoka, C. J. B. Ford, C. H. W. Barnes, D. Anderson, G. A. C. Jones, I. Farrer, and D. A. Ritchie, On-Demand Single-Electron Transfer between Distant Quantum Dots, Nature (London) 477, 439 (2011).

[15] C. J. B. Ford, Transporting and Manipulating Single Electrons in Surface-Acoustic-Wave Minima, Phys. Status Solidi B 254, 1600658 (2017).

[16] M. M. de Lima and P. V. Santos, Modulation of Photonic Structures by Surface Acoustic Waves, Rep. Prog. Phys. 68, 1639 (2005).

[17] M. M. de Lima, F. Alsina, W. Seidel, and P. V. Santos, Focusing of Surface-Acoustic-Wave Fields on (100) GaAs Surfaces, J. Appl. Phys. 94, 7848 (2003).

[18] X. Ding et al., Surface Acoustic Wave Microfluidics, Lab Chip 13, 3626 (2013).

[19] D. Morgan, Surface Acoustic Wave Filters (Academic Press, Boston, 2007).

[20] S. Datta, Surface Acoustic Wave Devices (Prentice-Hall, Upper Saddle River, NJ, 1986).

[21] E. A. Cerda-Mendez, D. N. Krizhanovskii, M. Wouters, R. Bradley, K. Biermann, K. Guda, R. Hey, P. V. Santos, D. Sarkar, and M.S. Skolnick, Polariton Condensation in Dynamic Acoustic Lattices, Phys. Rev. Lett. 105, 116402 (2010). 
[22] I. V. Kukushkin, J. H. Smet, L. Höppel, U. Waizmann, M. Riek, W. Wegschneider, and K. von Klitzing, UltrahighFrequency Surface Acoustic Waves for Finite Wave-Vector Spectroscopy of Two-Dimensional Electrons, Appl. Phys. Lett. 85, 4526 (2004).

[23] T. Byrnes, P. Recher, N. Y. Kim, S. Utsunomiya, and Y. Yamamoto, Quantum Simulator for the Hubbard Model with Long-Range Coulomb Interactions Using Surface Acoustic Waves, Phys. Rev. Lett. 99, 016405 (2007).

[24] S. Büyükköse, B. Vratzov, D. Atac, J. van der Veen, P. V. Santos, and W. G. van der Wiel, Ultrahigh-Frequency Surface Acoustic Wave Transducers on $\mathrm{ZnO} / \mathrm{SiO}_{2} / \mathrm{Si}$ Using Nanoimprint Lithography, Nanotechnology 23, 315303 (2012).

[25] A. R. Hutson and D. L. White, Elastic Wave Propagation in Piezoelectric Semiconductors, J. Appl. Phys. 33, 40 (1962).

[26] P. Bierbaum, Interaction of Ultrasonic Surface Waves with Conduction Electrons in Thin Metal Films, Appl. Phys. Lett. 21, 595 (1972).

[27] A. Wixforth, J. P. Kotthaus, and G. Weimann, Quantum Oscillations in the Surface-Acoustic-Wave Attenuation Caused by a Two-Dimensional Electron System, Phys. Rev. Lett. 56, 2104 (1986).

[28] A. Wixforth, J. Scriba, M. Wassermeier, J. P. Kotthaus, G. Weimann, and W. Schlapp, Surface Acoustic Waves on GaAs $/ \mathrm{Al}_{x} \mathrm{Ga}_{1-x}$ As Heterostructures, Phys. Rev. B 40, 7874 (1989).

[29] W. Paul, Electromagnetic Traps for Charged and Neutral Particles, Rev. Mod. Phys. 62, 531 (1990).

[30] D. Leibfried, R. Blatt, C. Monroe, and D. Wineland, Quantum Dynamics of Single Trapped Ions, Rev. Mod. Phys. 75, 281 (2003).

[31] S. Rahav, I. Gilary, and S. Fishman, Time Independent Description of Rapidly Oscillating Potentials, Phys. Rev. Lett. 91, 110404 (2003).

[32] S. Rahav, I. Gilary, and S. Fishman, Effective Hamiltonians for Periodically Driven Systems, Phys. Rev. A 68, 013820 (2003).

[33] J. I. Cirac, L. J. Garay, R. Blatt, A. S. Parkins, and P. Zoller, Laser Cooling of Trapped Ions: The Influence of Micromotion, Phys. Rev. A 49, 421 (1994).

[34] S. Kohler, T. Dittrich, and P. Hänggi, Floquet-Markovian Description of the Parametrically Driven, Dissipative Harmonic Quantum Oscillator, Phys. Rev. E 55, 300 (1997).

[35] T. Fujisawa, T. H. Oosterkamp, W. G. van der Wiel, B. W. Broer, R. Aguado, S. Tarucha, and L. P. Kouwenhoven, Spontaneous Emission Spectrum in Double Quantum Dot Devices, Science 282, 932 (1998).

[36] T. Fujisawa, D. G. Austing, Y. Tokura, Y. Hirayama, and S. Tarucha, Allowed and Forbidden Transitions in Artificial Hydrogen and Helium Atoms, Nature (London) 419, 278 (2002).

[37] T. Hayashi, T. Fujisawa, H. D. Cheong, Y. H. Jeong, and Y. Hirayama, Coherent Manipulation of Electronic States in a Double Quantum Dot, Phys. Rev. Lett. 91, 226804 (2003).

[38] J. R. Petta, A. C. Johnson, C. M. Marcus, M. P. Hanson, and A. C. Gossard, Manipulation of a Single Charge in a Double Quantum Dot, Phys. Rev. Lett. 93, 186802 (2004).
[39] P. Barthelemy and L. M. K. Vandersypen, Quantum Dot Systems: A Versatile Platform for Quantum Simulations, Ann. Phys. (Berlin) 525, 808 (2013).

[40] V. Kornich, C. Kloeffel, and D. Loss, Phonon-Mediated Decay of Singlet-Triplet Qubits in Double Quantum Dots, Phys. Rev. B 89, 085410 (2014).

[41] K. Wang, C. Payette, Y. Dovzhenko, P. W. Deelman, and J.R. Petta, Charge Relaxation in a Single Electron Si/SiGe Double Quantum Dot, Phys. Rev. Lett. 111, 046801 (2013).

[42] J. G. Rodriguez-Madrid, G. F. Iriarte, J. Pedros, O. A. Williams, D. Brink, and F. Calle, Super-High-Frequency SAW Resonators on AlN/Diamond, IEEE Electron Device Lett. 33, 495 (2012).

[43] M. Benetti, D. Cannata, F. Di Pietrantonio, and E. Verona, Growth of AlN Piezoelectric Film on Diamond for HighFrequency Surface Acoustic Wave Devices, IEEE Trans. Ultrason. Ferroelectr. Freq. Control 52, 1806 (2005).

[44] M. B. Assouar, O. Elmazria, P. Kirsch, P. Alnot, V. Mortet, and C. Tiusan, High-Frequency Surface Acoustic Wave Devices Based on AlN/Diamond Layered Structure Realized Using e-Beam Lithography, J. Appl. Phys. 101, 114507 (2007).

[45] E. Glushkov, N. Glushkova, and C. Zhang, Surface and Pseudo-Surface Acoustic Waves Piezoelectrically Excited in Diamond-Based Structures, J. Appl. Phys. 112, 064911 (2012).

[46] M. Benetti, D. Cannata, F. Di Pietrantonio, V. I. Fedosov, and E. Verona, Gigahertz-Range Electro-Acoustic Devices Based on Pseudo-Surface-Acoustic Waves in AlN/ Diamond/Si Structures, Appl. Phys. Lett. 87, 033504 (2005).

[47] R. H. Blick, M. L. Roukes, W. Wegscheider, and M. Bichler, Freely Suspended Two-Dimensional Electron Gases, Physica (Amsterdam) 249B, 784 (1998).

[48] COMSOL Multiphysics ${ }^{\circledR}$ v. 5.2, COMSOL AB, Stockholm, Sweden, www.comsol.com.

[49] In our COMSOL simulations we neglect the presence of the thin $\mathrm{Al}_{x} \mathrm{Ga}_{1-x}$ As crystal layer with typically $x \approx 0.3$. As argued in Ref. [50], this treatment is approximately correct since the relevant material properties (elastic constants, densities, and dielectric constants) of $\mathrm{Al}_{x} \mathrm{Ga}_{1-x} \mathrm{As}$ and GaAs are very similar. The mode functions and speed of sound are largely defined by the elastic constants, which are roughly the same for both $\mathrm{Al}_{x} \mathrm{Ga}_{1-x}$ As and pure GaAs; for example, the speed of the Rayleigh SAW mode for $\mathrm{Al}_{0.3} \mathrm{Ga}_{0.7} \mathrm{As}$ is $v_{s} \approx 3010 \mathrm{~m} / \mathrm{s}$, which differs from that of pure GaAs by only $\sim 5 \%$. Also, the piezoelectric coupling constants are rather similar, with $e_{14} \approx 0.15 \mathrm{c} / \mathrm{m}^{2}$ for pure GaAs and $e_{14} \approx 0.145 \mathrm{c} / \mathrm{m}^{2}$ for $\mathrm{Al}_{0.3} \mathrm{Ga}_{0.7} \mathrm{As}$ [50].

[50] S. H. Simon, Coupling of Surface Acoustic Waves to a Two-Dimensional Electron Gas, Phys. Rev. B 54, 13878 (1996).

[51] K. S. Novoselov, A. Mishchenko, A. Carvalho, and A. H. Castro Neto, 2D Materials and van der Waals Heterostructures, Science 353, aac9439 (2016).

[52] A. Kormanyos, G. Burkard, M. Gmitra, J. Fabian, V. Zolyomi, N. D. Drummond, and V. Fal'ko, $k \cdot p$ Theory for Two-Dimensional Transition Metal Dichalcogenide Semiconductors, 2D Mater. 2, 022001 (2015). 
[53] T. Eknapakul et al., Electronic Structure of a QuasiFreestanding $\mathrm{Mos}_{2}$ Monolayer, Nano Lett. 14, 1312 (2014).

[54] K. F. Mak, K. He, C. Lee, G. H. Lee, J. Hone, T. F. Heinz, and J. Shan, Tightly Bound Trions in Monolayer $\mathrm{Mos}_{2}$, Nat. Mater. 12, 207 (2013).

[55] J. S. Ross et al., Electrical Control of Neutral and Charged Excitons in a Monolayer Semiconductor, Nat. Commun. 4, 1474 (2013).

[56] P. H. Dawson and Y. Bingqi, The Second Stability Region of the Quadrupole Mass Filter. I. Ion Optical Properties, Int. J. Mass Spectrom. Ion Process. 56, 25 (1984).

[57] P. H. Dawson and Y. Bingqi, The Second Stability Region of the Quadrupole Mass Filter. II. Experimental Results, Int. J. Mass Spectrom. Ion Process. 56, 41 (1984).

[58] F. J. R. Schülein, E. Zallo, P. Atkinson, O. G. Schmidt, R. Trotta, A. Rastelli, A. Wixforth, and H. J. Krenner, Fourier Synthesis of Radio Frequency Nanomechanical Pulses with Different Shapes, Nat. Nanotechnol. 10, 512 (2015).

[59] G. C. Possa and L. F. Roncaratti, Stability Diagrams for Paul Ion Traps Driven by Two Frequencies, J. Phys. Chem. A 120, 4915 (2016).

[60] Here and we restrict our discussion to the pseudopotential regime where $q^{2} \ll 1$, as done in the major body of our work (apart from the discussion of exotic stability regions where the heating-related arguments should be contrasted with an increased temperature robustness).

[61] S. Furuta, C. H. W. Barnes, and C. J. L. Doran, Single-Qubit Gates and Measurements in the Surface Acoustic Wave Quantum Computer, Phys. Rev. B 70, 205320 (2004).

[62] F. Martins, F. K. Malinowski, P. D. Nissen, E. Barnes, S. Fallahi, G. C. Gardner, M. J. Manfra, C. M. Marcus, and F. Kuemmeth, Noise Suppression Using Symmetric Exchange Gates in Spin Qubits, Phys. Rev. Lett. 116, 116801 (2016).

[63] M. D. Reed et al., Reduced Sensitivity to Charge Noise in Semiconductor Spin Qubits via Symmetric Operation, Phys. Rev. Lett. 116, 110402 (2016).

[64] T. A. Baart, T. Fujita, C. Reichl, W. Wegscheider, and L. M. K. Vandersypen, Coherent Spin-Exchange via a Quantum Mediator, Nat. Nanotechnol. 12, 26 (2017).

[65] C. Campbell, Surface Acoustic Wave Devices for Mobile and Wireless Communications (Academic Press, New York, 1998).

[66] D. Jaksch, C. Bruder, J. I. Cirac, C. W. Gardiner, and P. Zoller, Cold Bosonic Atoms in Optical Lattices, Phys. Rev. Lett. 81, 3108 (1998).

[67] W. Hofstetter, J. I. Cirac, P. Zoller, E. Demler, and M. D. Lukin, High-Temperature Superfluidity of Fermionic Atoms in Optical Lattices, Phys. Rev. Lett. 89, 220407 (2002).

[68] P.W. Anderson, Model for the Electronic Structure of Amorphous Semiconductors, Phys. Rev. Lett. 34, 953 (1975).

[69] A. Singha et al., Two-Dimensional Mott-Hubbard Electrons in an Artificial Honeycomb Lattice, Science 332, 1176 (2011).

[70] T. Schlösser et al., Internal Structure of a Landau Band Induced by a Lateral Superlattice: A Glimpse of Hofstadter's Butterfly, Europhys. Lett. 33, 683 (1996).

[71] C. Albrecht, J. H. Smet, K. von Klitzing, D. Weiss, V. Umansky, and H. Schweizer, Evidence of Hofstadters
Fractal Energy Spectrum in the Quantized Hall Conductance, Phys. Rev. Lett. 86, 147 (2001).

[72] T. Hensgens, T. Fujita, L. Janssen, X. Li, C. J. Van Diepen, C. Reichl, W. Wegscheider, S. Das Sarma, and L. M. K. Vandersypen, Quantum Simulation of a Fermi-Hubbard Model Using a Semiconductor Quantum Dot Array, Nature (London) 548, 70 (2017).

[73] I. Kuljanishvili, C. Kayis, J. F. Harrison, C. Piermarocchi, T. A. Kaplan, S. H. Tessmer, L. N. Pfeiffer, and K. W. West, Scanning-Probe Spectroscopy of Semiconductor Donor Molecules, Nat. Phys. 4, 227 (2008).

[74] J. Martin, S. Ilani, B. Verdene, J. Smet, V. Umansky, D. Mahalu, D. Schuh, G. Abstreiter, and A. Yacoby, Localization of Fractionally Charged Quasi-Particles, Science 305, 980 (2004).

[75] O. E. Dial, R. C. Ashoori, L. N. Pfeiffer, and K. W. West, High-Resolution Spectroscopy of Two-Dimensional Electron Systems, Nature (London) 448, 176 (2007).

[76] O. Gywat, H. J. Krenner, and J. Berezovsky, Spins in Optically Active Quantum Dots (Wiley-VCH, Berlin, 2010).

[77] A. Srivastava, M. Sidler, A. V. Allain, D. S. Lembke, A. Kis, and A. Imamoglu, Optically Active Quantum Dots in Monolayer $\mathrm{Mos}_{2}$, Nat. Nanotechnol. 10, 491 (2015).

[78] A. N. Vamivakas, Y. Zhao, C.-Y. Lu, and M. Atatüre, SpinResolved Quantum-Dot Resonance Fluorescence, Nat. Phys. 5, 198 (2009).

[79] M. Atatüre, J. Dreiser, A. Badolato, and A. Imamoglu, Observation of Faraday Rotation from a Single Confined Spin, Nat. Phys. 3, 101 (2007).

[80] J. Berezovsky, M. H. Mikkelsen, O. Gywat, N. G. Stoltz, L. A. Coldren, and D. D. Awschalom, Nondestructive Optical Measurements of a Single Electron Spin in a Quantum Dot, Science 314, 1916 (2006).

[81] T. A. Baart, M. Shafiei, T. Fujita, C. Reichl, W. Wegscheider, and L. M. K. Vandersypen, Single-Spin CCD, Nat. Nanotechnol. 11, 330 (2016).

[82] T. Byrnes, N. Y. Kim, K. Kusudo, and Y. Yamamoto, Quantum Simulation of Fermi-Hubbard Models in Semiconductor Quantum-Dot Arrays, Phys. Rev. B 78, 075320 (2008).

[83] Here, we take the mobility $\mu$ as our figure of merit, as it is one of the standard metrics to characterize the effect of disorder. Both long-range scattering and short-range scattering are reflected in the mobility, and in fact, the exact dependence of mobility on density often allows one to distinguish whether long-range or short-range scattering dominates. The main caveat about using mobility is that it is typically measured in density ranges larger than those used in the present experiments. The same caveat, however, applies to the use of mobility for predicting quantum dot behavior. Yet, empirically, for quantum dots defined in systems with relatively low mobility, such as $\mathrm{Si} / \mathrm{SiGe}$ quantum wells, mobility is found to be a good predictor of the ability to realize well-behaved quantum dots, i.e., not suffering excessive disorder [84]. For this reason, and given that mobility has been measured across many systems, we refer to mobility as an indication of disorder.

[84] F. A. Zwanenburg, A. S. Dzurak, A. Morello, M. Y. Simmons, L. C. L. Hollenberg, G. Klimeck, S. Rogge, 
S. N. Coppersmith, and M. A. Eriksson, Silicon Quantum Electronics, Rev. Mod. Phys. 85, 961 (2013).

[85] V. Umansky, R. de-Picciotto, and M. Heiblum, Extremely High-Mobility Two Dimensional Electron Gas: Evaluation of Scattering Mechanisms, Appl. Phys. Lett. 71, 683 (1997).

[86] D. Hofstadter, Energy Levels and Wave Functions of Bloch Electrons in Rational and Irrational Magnetic Fields, Phys. Rev. B 14, 2239 (1976).

[87] A. Lagendijk, B. A. Van Tiggelen, and D. Wiersma, Fifty Years of Anderson Localization, Phys. Today 62, No. 8, 24 (2009).

[88] D. Belitz and T.R. Kirkpatrick, The Anderson-Mott Transition, Rev. Mod. Phys. 66, 261 (1994).

[89] D. Basko, I. Aleiner, and B. Altshuler, Metal-Insulator Transition in a Weakly Interacting Many-Electron System with Localized Single-Particle States, Ann. Phys. (Amsterdam) 321, 1126 (2006).

[90] K. Byczuk, W. Hofstetter, and D. Vollhardt, Mott-Hubbard Transition versus Anderson Localization in Correlated Electron Systems with Disorder, Phys. Rev. Lett. 94, 056404 (2005).

[91] L. Fallani, J. E. Lye, V. Guarrera, C. Fort, and M. Inguscio, Ultracold Atoms in a Disordered Crystal of Light: Towards a Bose Glass, Phys. Rev. Lett. 98, 130404 (2007).

[92] O. P. Sushkov and A. H. Castro Neto, Topological Insulating States in Laterally Patterned Ordinary Semiconductors, Phys. Rev. Lett. 110, 186601 (2013).

[93] J. V. T. Buller, R. E. Balderas-Navarro, K. Biermann, E. A. Cerda-Mendez, and P. V. Santos, Exciton-Polariton Gap Soliton Dynamics in Moving Acoustic Square Lattices, Phys. Rev. B 94, 125432 (2016).

[94] L. V. Butov et al., Condensation and Pattern Formation in Cold Exciton Gases in Coupled Quantum Wells, J. Phys. Condens. Matter 16, R1577 (2004).

[95] A. T. Hammack, M. Griswold, L. V. Butov, L. E. Smallwood, A. L. Ivanov, and A. C. Gossard, Trapping of Cold Excitons with Laser Light, Phys. Rev. Lett. 96, 227402 (2006).

[96] A. T. Hammack, L. V. Butov, L. Mouchliadis, A. L. Ivanov, and A. C. Gossard, Kinetics of Indirect Excitons in an Optically Induced Trap in GaAs Quantum Wells, Phys. Rev. B 76, 193308 (2007).

[97] S. Kumar, A. Kaczmarczyk, and B. D. Gerardot, StrainInduced Spatial and Spectral Isolation of Quantum Emitters in Mono- and Bilayer Mos 2 , Nano Lett. 15, 7567 (2015).

[98] M. J. A. Schuetz, E. M. Kessler, G. Giedke, L. M. K. Vandersypen, M. D. Lukin, and J. I. Cirac, Universal Quantum Transducers Based on Surface Acoustic Waves, Phys. Rev. X 5, 031031 (2015).

[99] J.-X. Liu, D.-N. Fang, W.-Y. Wei, and X.-F. Zhao, Love Waves in Layered Piezoelectric/Piezomagnetic Structures, J. Sound Vib. 315, 146 (2008).
[100] Y. Pang, J.-X. Liu, Y.-S. Wang, and X.-F. Zhang, Propagation of Rayleigh-Type Surface Waves in a Transversely Isotropic Piezoelectric Layer on a Piezomagnetic HalfSpace, J. Appl. Phys. 103, 074901 (2008).

[101] M. Weiler, L. Dreher, C. Heeg, H. Huebl, R. Gross, M. S. Brandt, and S. T. B. Goennenwein, Acoustically Driven Ferromagnetic Resonance, Phys. Rev. Lett. 106, 117601 (2011).

[102] J. Knörzer et al. (to be published).

[103] R. J. Glauber, in Laser Manipulation of Atoms and Ions, Proceedings of the International School of Physics "Enrico Fermi," Course 118, edited by E. Arimondo, W. D. Phillips, and F. Strumia (North-Holland, Amsterdam, 1992), p. 643.

[104] V. N. Golovach, A. Khaetskii, and D. Loss, PhononInduced Decay of the Electron Spin in Quantum Dots, Phys. Rev. Lett. 93, 016601 (2004).

[105] H.-P. Breuer and F. Petruccione, The Theory of Open Quantum Systems (Oxford University Press, Oxford, 2002).

[106] R. J. Schnebele, M. Kataoka, C. J. B. Ford, C. H. W. Barnes, D. Anderson, G. A. C. Jones, I. Farrer, D. A. Ritchie, and M. Pepper, Quantum-Dot Thermometry of Electron Heating by Surface Acoustic Waves, Appl. Phys. Lett. 89, 122104 (2006).

[107] P. Utko, P. E. Lindelof, and K. Gloos, Heating in SingleElectron Pumps Driven by Surface Acoustic Waves, Appl. Phys. Lett. 88, 202113 (2006).

[108] R. Manenti, M. J. Peterer, A. Nersisyan, E. B. Magnusson, A. Patterson, and P. J. Leek, Surface Acoustic Wave Resonators in the Quantum Regime, Phys. Rev. B 93, 041411(R) (2016).

[109] W. Wu et al., Piezoelectricity of Single-Atomic-Layer $\mathrm{MoS}_{2}$ for Energy Conversion and Piezotronics, Nature (London) 514, 470 (2014).

[110] M. K. Ekstroem, T. Aref, J. Runeson, J. Bjoerck, I. Bostroem, and P. Delsing, Surface Acoustic Wave Unidirectional Transducers for Quantum Applications, Appl. Phys. Lett. 110, 073105 (2017).

[111] L. M. K. Vandersypen, H. Bluhm, J. S. Clarke, A. S. Dzurak, R. Ishihara, A. Morello, D. J. Reilly, L. R. Schreiber, and M. Veldhorst, Interfacing Spin Qubits in Quantum Dots and Donors-Hot, Dense and Coherent, arXiv:1612.05936.

[112] E. A. Chekhovich, M. N. Makhonin, A. I. Tartakovskii, A. Yacoby, H. Bluhm, K. C. Nowack, and L. M. K. Vandersypen, Nuclear Spin Effects in Semiconductor Quantum Dots, Nat. Mater. 12, 494 (2013).

[113] M. Veldhorst et al., An Addressable Quantum Dot Qubit with Fault-Tolerant Control Fidelity, Nat. Nanotechnol. 9, 981 (2014). 